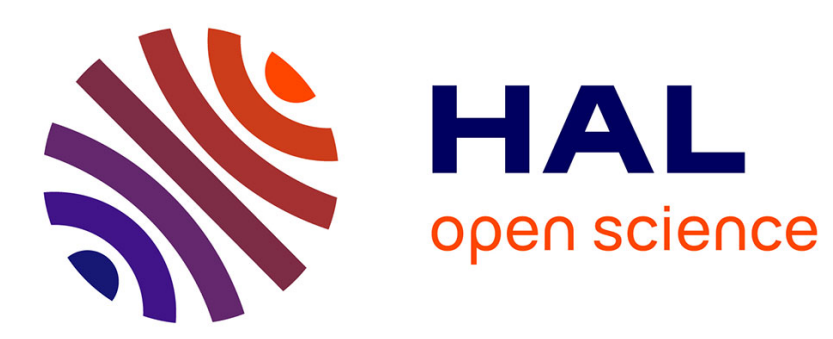

\title{
Symbolic verification of privacy-type properties for security protocols with XOR
}

David Baelde, Stéphanie Delaune, Ivan Gazeau, Steve Kremer

\section{To cite this version:}

David Baelde, Stéphanie Delaune, Ivan Gazeau, Steve Kremer. Symbolic verification of privacy-type properties for security protocols with XOR. CSF 2017 - 30th IEEE Computer Security Foundations Symposium, Aug 2017, Santa Barbara, United States. pp.15. hal-01533708

\section{HAL Id: hal-01533708 \\ https://hal.inria.fr/hal-01533708}

Submitted on 6 Jun 2017

HAL is a multi-disciplinary open access archive for the deposit and dissemination of scientific research documents, whether they are published or not. The documents may come from teaching and research institutions in France or abroad, or from public or private research centers.
L'archive ouverte pluridisciplinaire HAL, est destinée au dépôt et à la diffusion de documents scientifiques de niveau recherche, publiés ou non, émanant des établissements d'enseignement et de recherche français ou étrangers, des laboratoires publics ou privés. 


\title{
Symbolic verification of privacy-type properties for security protocols with XOR
}

\author{
David Baelde*, Stéphanie Delaune*†, Ivan Gazeau* ${ }^{* \ddagger}$, and Steve Kremer ${ }^{\ddagger}$ \\ ${ }^{*}$ LSV, ENS Cachan \& CNRS \& INRIA Saclay Île-de-France, France \\ ${ }^{\dagger}$ CNRS \& IRISA, Rennes, France \\ ${ }_{\ddagger}^{\ddagger}$ LORIA, INRIA Nancy - Grand-Est, France
}

\begin{abstract}
In symbolic verification of security protocols, process equivalences have recently been used extensively to model strong secrecy, anonymity and unlinkability properties. However, tool support for automated analysis of equivalence properties is limited compared to trace properties, e.g., modeling authentication and weak notions of secrecy. In this paper, we present a novel procedure for verifying equivalences on finite processes, i.e., without replication, for protocols that rely on various cryptographic primitives including exclusive or (xor). We have implemented our procedure in the tool AKISS, and successfully used it on several case studies that are outside the scope of existing tools, e.g., unlinkability on various RFID protocols, and resistance against guessing attacks on protocols that use xor.
\end{abstract}

\section{INTRODUCTION}

Protecting authenticity and confidentiality of transactions by the use of cryptography has become standard practice. Security protocols such as TLS, SSH, or Kerberos are nowadays widely deployed. However, history has shown that designing secure protocols is challenging, because of the concurrent execution of protocols in an adversarial environment. Many attacks exploit flaws in the protocol logic rather than, or sometimes combined with, weaknesses in the underlying cryptographic primitives, e.g., [14]. During the past two decades, several efficient automated verification tools have been developed to detect logical flaws, e.g., ProVerif [17], AVISPA [6], MaudeNPA [29], Tamarin [39], and they have successfully discovered many attacks in academic protocols [35], standards [11] and deployed protocols [5].

Authentication and (weak forms of) confidentiality are modelled as trace properties: they are checked by verifying that each possible trace of the system satisfies some predicate. The verification of such properties is nowadays well understood and enjoys efficient tool support, as discussed above. However, some properties such as resistance against guessing attacks, strong secrecy, anonymity and unlinkability [25], [28], [4], [18] are expressed in terms of indistinguishability, a form of process equivalence [2], [1]. Automated verification of equivalence properties is not yet as mature as for trace properties. Several tools have recently been extended with the possibility to verify a strong equivalence called diff-equivalence [16],

The research leading to these results has received funding from the European Research Council (ERC) under the European Union's Horizon 2020 research and innovation program (grant agreements No 645865-SPOOC and No 714955-POPSTAR), as well as from the French National Research Agency (ANR) under the project JCJC VIP n ${ }^{\circ} 11$ JS02 00601 and the project Sequoia.
[12], [38]. This equivalence can only relate processes that only differ in the messages that they use, but not in their control flow, which is too strong for some applications. Dedicated tools for verifying process equivalences on security protocols, in the case of a bounded number of sessions, have also been developed. The SPEC tool [40] decides a symbolic bisimulation, which implies trace equivalence, for protocols that use a fixed set of standard cryptographic primitives. It does not support protocols with else branches. The APTE tool [20] decides trace equivalence for processes including else branches, and a fixed set of standard primitives. The AKISS tool [19] is able to check trace equivalence for processes without else branches but supports various cryptographic primitives, including most standard ones as well as, e.g., blind signatures and trapdoor bit commitment.

Some protocols use cryptographic primitives that have algebraic properties [26]. Exclusive or (xor) is such a primitive, and protocols implemented on low-power devices, such as RFID tags, often rely on it because of its computational efficiency [42]. There exist many results for taking into account algebraic properties for trace based properties, in particular for the xor operator [24], [22], [34]. However, only a few procedures for equivalence properties take algebraic properties into account. Delaune et al. [27] have studied equivalence of constraint systems, showing in particular that the theory for xor is decidable in PTIME. However, they only consider the case of pure group theories, not allowing any other equations, e.g., those modelling encryption. When considering an unbounded number of sessions, tools that can verify diffequivalence do not effectively support xor. The Tamarin tool supports a theory for Diffie-Hellman exponentiations, but not xor. The Maude-NPA tool supports xor in principle, but when verifying equivalence properties it does not terminate even on simple examples. For trace properties such as secrecy and authentication, termination is achieved in practice on several examples, e.g., a xor based variant of the NSL protocol, the IBM CCA crypto API: sometimes, attacks are discovered by bounding the search depth to avoid non-termination; the analysis of CCA also relies on Never Patterns, a technique that allows to prune the search space, but which requires an external, protocol-dependent [33] justification.

AKISS in a nutshell: The procedure that we present in this paper builds on previous work by Chadha et al. [19], 
and its implementation in the AKISS tool. This tool checks equivalences for protocols modelled as processes in a calculus similar to the applied pi-calculus [1], but without else branches nor replication. It actually checks for two equivalences which over- and under-approximate the standard notion of trace equivalence for cryptographic protocols, allowing one to either prove or disprove trace equivalence. The coarser equivalence also coincides with trace equivalence on the large class of determinate processes.

The AKISS tool supports cryptographic primitives that can be expressed through a convergent rewrite system enjoying the finite variant property [23]. Termination is guaranteed for the subclass of subterm convergent rewrite theories, but also achieved in practice on several examples outside this class.

The procedure is based on a fully abstract modelling of symbolic traces of the protocols into first-order Horn clauses. Each symbolic trace is translated into a set of clauses called seed statements, and a dedicated resolution procedure is applied to this set to construct a set of statements that have a simple form, called solved statements. It is shown that these solved statements form a sound and complete representation of the symbolic trace under study. Therefore, only the set of solved statements is required to decide whether a given symbolic trace is included in some process. To decide trace equivalence between processes $P$ and $Q$, the procedure checks whether each symbolic trace of $P$ is included in $Q$ as described above, and vice versa.

Contributions: We design a new procedure for verifying trace equivalence of protocols that use the xor operator, extending the work by Chadha et al. [19]. Our procedure follows the general structure of the original one, modelling protocols as processes in (a variant of the) applied pi-calculus (Section II) and translating symbolic traces into Horn clauses (Section III). The xor operator is not supported in AKISS because it cannot be modelled by a convergent rewrite system. Our approach consists in first orienting the equations of xor into a convergent rewrite system modulo associativity and commutativity $(\mathrm{AC})$, then generalizing the procedure of Chadha et al. to reason modulo AC (Section IV). A direct generalisation would be sound and complete, but would not terminate even on very simple examples. We therefore completely redesign the resolution procedure, using a new strategy to forbid certain steps that would yield non-termination. Showing that these steps are indeed unnecessary requires essential changes to the completeness proof. The modified procedure yields an effective algorithm for checking trace equivalence (Section V). Although termination is not guaranteed, we have implemented our procedure as an extension of AKISS and demonstrated its effectiveness on several examples, including unlinkability for various RFID protocols [42] and resistance against guessing attacks for password based protocols [31], [32]. To the best of our knowledge, our tool is the first that can effectively verify equivalence properties for protocols that use xor. As with all of the above-mentionned tools, our results are restricted to the (symbolic) model under consideration: when our algorithm concludes that there is no attack, it is only meaningful in that model. A proof in the computational model would be stronger, but would still bear some limitations. A natural question is whether our symbolic model could be computationally sound. Note that the impossibily result of [41] does not apply here, since we only consider bounded runs. Nevertheless, we do not claim computational soundness, and argue instead that symbolic security proofs are already useful information.

Outline: In Section II, we introduce our formalism for modelling protocols, a variant of the replication-free fragment of the applied pi calculus. In Section III] we provide a fully abstract representation in Horn clauses of protocols expressed in our calculus. Next, we present our saturation procedure based on Horn clause resolution in Section IV. We present the algorithm for checking trace equivalence, its implementation, and our case studies in Section V. Full proofs are available in the long version of this paper [8].

\section{PRocess CALCULUS}

We introduce the process calculus and the notion of equivalence that we use to model protocols and indistinguishability. Our calculus has similarities with the applied pi-calculus [1] which has been extensively used to specify security protocols. Participants in a protocol are modeled as processes, and the communication between them is modeled by means of message passing.

\section{A. Term algebra}

As usual in symbolic models we model messages as terms. We consider several sets of atomic terms:

- $\mathcal{N}$ is a set of names, partitioned into the disjoint sets $\mathcal{N}_{\text {prv }}$ and $\mathcal{N}_{\text {pub }}$ of private and public names;

- $\mathcal{X}$ is the set of message variables, denoted $x, y$, etc.;

- $\mathcal{W}=\left\{\mathrm{w}_{1}, \mathrm{w}_{2}, \ldots\right\}$ is the set of parameters.

Intuitively, private names in $\mathcal{N}_{\text {prv }}$ represent nonces or keys generated by honest participants, while public names in $\mathcal{N}_{\text {pub }}$ represent identifiers available both to the attacker and to honest participants, and attacker nonces. Parameters are used by the attacker as pointers to refer to messages that were previously output by the protocol participants.

Given a signature $\Sigma$ (i.e., a finite set of function symbols together with their arity) and a set of atoms $\mathcal{A}$ we denote by $\mathcal{T}(\Sigma, \mathcal{A})$ the set of terms, defined as the smallest set that contains $\mathcal{A}$ and that is closed under application of function symbols in $\Sigma$. We denote by vars $(t)$ the set of variables occurring in a term $t$. As usual, a substitution is a function from variables to terms, that is lifted to terms homomorphically. The application of a substitution $\sigma$ to a term $u$ is written $u \sigma$, and we denote $\operatorname{dom}(\sigma)$ its domain, i.e. $\operatorname{dom}(\sigma)=\{x \mid \sigma(x) \neq x\}$. The identity substitution, of empty domain, is noted $\emptyset$. The positions of a term are defined as usual.

We associate an equational theory $\mathrm{E}$ to the signature $\Sigma$. It consists of a finite set of equations of the form $M=N$ where $M, N \in \mathcal{T}(\Sigma, \mathcal{X})$, and induces an equivalence relation 
over terms: $=_{\mathrm{E}}$ is the smallest congruence on terms, which contains all equations $M=N$ in $\mathrm{E}$, and that is closed under substitution of terms for variables. To model protocols that only rely on the xor operator, we consider $\Sigma_{\text {xor }}=\{\oplus, 0\}$, and the equational theory $\mathrm{E}_{\mathrm{xor}}$ below:

$$
\begin{array}{rlrlrl}
x \oplus x & =0 & x \oplus(y \oplus z) & = & (x \oplus y) \oplus z \\
x \oplus 0 & =x & x \oplus y & =y \oplus x
\end{array}
$$

We denote by $\mathrm{AC}$ the equational theory defined by the two equations on the right. We may also want to consider additional primitives, e.g. pairs, symmetric and asymmetric encryptions, signatures, hashes, etc. This can be done by extending the signature as well as the equational theory.

Example 1: Let $\Sigma_{\text {xor }}^{+}=\Sigma_{\text {xor }} \uplus\left\{\langle\cdot, \cdot\rangle, \operatorname{proj}_{1}, \operatorname{proj}_{2}, \mathrm{~h}\right\}$, and consider the equational theory $\mathrm{E}_{\text {xor }}^{+}$extending $\mathrm{E}_{\mathrm{xor}}$ with the equations $\operatorname{proj}_{1}(\langle x, y\rangle)=x$, and $\operatorname{proj}_{2}(\langle x, y\rangle)=y$. The symbol $\langle\cdot, \cdot\rangle$ models pairs; the $\operatorname{proj}_{i}$ symbols model projections; the unary symbol h models a hash function. Take $i d \in$ $\mathcal{N}_{\text {pub }}$ to model the identity of a participant, and $r_{1}, r_{2}, k \in \mathcal{N}_{\text {prv }}$ to represent two random numbers and a key, a priori unknown to the attacker. Let $t_{0}=\left\langle i d \oplus r_{2}, \mathrm{~h}\left(\left\langle r_{1}, k\right\rangle\right) \oplus r_{2}\right\rangle$. We have that $\left(\operatorname{proj}_{1}\left(t_{0}\right) \oplus i d\right) \oplus \operatorname{proj}_{2}\left(t_{0}\right)={ }_{E_{\text {xor }}^{+}} \mathrm{h}\left(\left\langle r_{1}, k\right\rangle\right)$.

In this paper we consider a signature $\Sigma$ such that $\Sigma_{\text {xor }} \subseteq \Sigma$, together with an equational theory generated by a set of equations of the form

$$
\mathrm{E}=\mathrm{E}_{\text {xor }} \cup\left\{M=N \mid M, N \in \mathcal{T}\left(\Sigma \backslash \Sigma_{\text {xor }}, \mathcal{X}\right)\right\} .
$$

Hence, E models xor in combination with any other equational theory that is disjoint from $E_{x o r}$.

\section{B. Finite variant property}

A rewrite system $\mathcal{R}$ is a set of rewrite rules of the form $\ell \rightarrow r$ where $\ell, r \in \mathcal{T}(\Sigma, \mathcal{X})$, and $\operatorname{vars}(r) \subseteq \operatorname{vars}(\ell)$. A term $t$ can be rewritten in one step (modulo AC) to $u$, denoted $t \rightarrow_{\mathcal{R}, \mathrm{AC}} u$, if there exists a position $p$ in term $t$, a rule $\ell \rightarrow r$ in $\mathcal{R}$ and a substitution $\sigma$ such that $\left.t\right|_{p}={ }_{\mathrm{AC}} \ell \sigma$ and $u=t[r \sigma]_{p}$, i.e., the term at position $p$ in $t$ is equal to $\ell \sigma$ modulo $A C$ and $u$ is the term obtained by replacing, in $t$, the subterm $\left.t\right|_{p}$ with $r \sigma$. The relation $\rightarrow_{\mathcal{R}, A C}^{*}$ denotes the transitive and reflexive closure of $\rightarrow_{\mathcal{R}, \mathrm{AC}}$.

A rewrite system $\mathcal{R}$ is AC-convergent if the relation $\rightarrow_{\mathcal{R}, A C}^{*}$ is confluent and strongly terminating. We denote by $t_{\downarrow_{\mathcal{R}, A C} \text { (or }}$ simply $t \downarrow$ ) the normal form of a term $t$. In the following we only consider equational theories $E$ that can be represented by a rewrite system $\mathcal{R}$ which is AC-convergent, i.e., such that

$$
u={ }_{\mathrm{E}} v \Leftrightarrow u \downarrow_{\mathcal{R}, \mathrm{AC}}={ }_{\mathrm{AC}} v \downarrow_{\mathcal{R}, \mathrm{AC}} .
$$

Example 2: The equational theory $\mathrm{E}_{\text {xor }}^{+}$of Example 1 can be represented by the following AC-convergent system:

$$
\mathcal{R}_{\text {xor }}^{+}=\left\{\begin{array}{cc}
x \oplus(x \oplus y) \rightarrow y & \operatorname{proj}_{1}(\langle x, y\rangle) \rightarrow x \\
x \oplus 0 \rightarrow x & \operatorname{proj}_{2}(\langle x, y\rangle) \rightarrow y \\
x \oplus x \rightarrow 0 &
\end{array}\right.
$$

Let $t_{0}=\left\langle i d \oplus r_{2}, \mathrm{~h}\left(\left\langle r_{1}, k\right\rangle\right) \oplus r_{2}\right\rangle$. We have that:

$$
\begin{array}{ll} 
& \operatorname{proj}_{1}\left(t_{0}\right) \oplus \operatorname{proj}_{2}\left(t_{0}\right) \\
\rightarrow_{\mathcal{R}_{\text {xor }}^{+}, \mathrm{AC}} & \left(i d \oplus r_{2}\right) \oplus \operatorname{proj}_{2}\left(t_{0}\right) \\
\rightarrow_{\mathcal{R}_{\text {xor }}^{+}, \mathrm{AC}} & \left(i d \oplus r_{2}\right) \oplus\left(\mathrm{h}\left(\left\langle r_{1}, k\right\rangle\right) \oplus r_{2}\right) \\
\rightarrow_{\mathcal{R}_{\text {xor }}^{+}, \mathrm{AC}} & i d \oplus \mathrm{h}\left(\left\langle r_{1}, k\right\rangle\right)
\end{array}
$$

Note that the first rule in $\mathcal{R}_{\text {xor }}^{+}$is essential in last rewriting step above, and more generally for $\mathcal{R}_{\text {xor }}^{+}$to represent $E_{\text {xor }}$.

Given an AC-convergent rewrite system $\mathcal{R}$, we define complete sets of variants, first introduced in [23].

Definition 1: Consider a rewrite system $\mathcal{R}$ that is ACconvergent, and a set of terms $T$. A set of substitutions variants $_{\mathcal{R}, \mathrm{AC}}(T)$ is called a complete set of variants for the set of terms $T$, if for any substitution $\omega$ there exist $\sigma \in$ variants $_{\mathcal{R}, \mathrm{AC}}(T)$, and a substitution $\tau$ such that:

- $x \omega \downarrow={ }_{\mathrm{AC}} x \sigma \downarrow \tau$ for any $x \in \operatorname{vars}(T)$, and

- $(t \omega) \downarrow={ }_{\mathrm{AC}}(t \sigma) \downarrow \tau$ for any $t \in T$.

The set of variants of $t$ represents a pre-computation such that the normal form of any instance of $t$ is equal (modulo AC) to an instance of $t \sigma \downarrow$ for some $\sigma$ in the set of variants, without the need to apply further rewrite steps. A rewrite system has the finite variant property if for any set of terms one can compute a finite complete set of variants. We will often write variants $\mathcal{R}_{\mathcal{R}, \mathrm{AC}}\left(t_{1}, \ldots, t_{n}\right)$ instead of $\operatorname{variants}_{\mathcal{R}, \mathrm{AC}}\left(\left\{t_{1}, \ldots, t_{n}\right\}\right)$.

Example 3: Considering the equational theory $\mathrm{E}_{\mathrm{xor}}^{+}$introduced in Example 11, and the rewrite system defined in Example 2 We have $\sigma=\left\{x \mapsto\left\langle x_{1}, x_{2}\right\rangle\right\} \in$ $\operatorname{variants}_{\mathcal{R}, \mathrm{AC}}\left(\operatorname{proj}_{1}(x)\right)$. Actually, $\sigma$ together with the identity substitution form a complete set of variants for $\operatorname{proj}_{1}(x)$.

The following substitutions, together with the identity substitution, form a complete set of variants for $x \oplus y$ :

$$
\begin{aligned}
& \text { - } \sigma_{1}=\{x \mapsto y \oplus z\}, \sigma_{1}^{\prime}=\{y \mapsto x \oplus z\}, \\
& \text { - } \sigma_{2}=\left\{x \mapsto x^{\prime} \oplus z, y \mapsto y^{\prime} \oplus z\right\}, \\
& \text { - } \sigma_{3}=\{y \mapsto x\}, \text { and } \\
& \text { - } \sigma_{4}=\{x \mapsto 0\}, \sigma_{4}^{\prime}=\{y \mapsto 0\} .
\end{aligned}
$$

This finite variant property is satisfied by many equational theories of interest, e.g., symmetric and asymmetric encryptions, signatures, blind signatures, zero-knowledge proofs. Moreover, such a property plays an important role regarding equational unification. It implies the existence of a complete set of unifiers, and gives us a way to compute it effectively [30].

Definition 2: Consider an AC-convergent $\mathcal{R}$ and a set of equations $\Gamma=\left\{u_{1}=v_{1}, \ldots, u_{k}=v_{k}\right\}$. A set of substitutions $S$ is a complete set of $\mathcal{R}, \mathrm{AC}$-unifiers for $\Gamma$ if:

1) for each $\sigma \in S$ and $i \in\{1, \ldots, k\}, u_{i} \sigma \downarrow={ }_{\mathrm{AC}} v_{i} \sigma \downarrow$;

2) for each $\theta$ such that $u_{i} \theta \downarrow={ }_{\mathrm{AC}} v_{i} \theta \downarrow$ for all $i \in\{1, \ldots, k\}$, there exists $\sigma \in S$ and a substitution $\tau$ such that $x \theta \downarrow=\mathrm{AC}$ $x \sigma \tau \downarrow$ for any $x \in \operatorname{vars}(\Gamma)$.

Such a set is denoted $\operatorname{csu}_{\mathcal{R}, \mathrm{AC}}(\Gamma)$, or $\operatorname{csu}_{\mathrm{AC}}(\Gamma)$ when $\mathcal{R}=\emptyset$. 


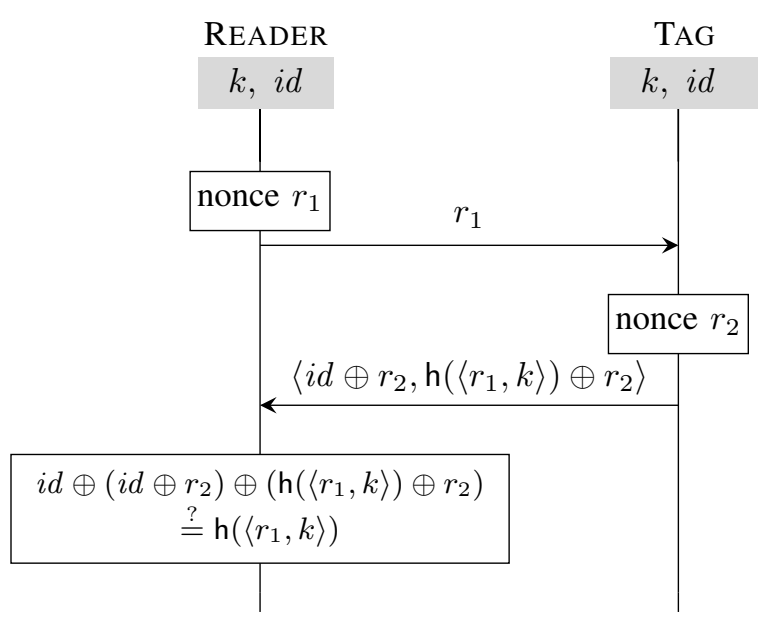

Fig. 1: The KCL protocol

\section{Process calculus}

Let $\mathcal{C} h$ be a set of public channels. A protocol is modeled by a finite set of processes generated by the following grammar:

$$
\begin{array}{rlr}
P, P^{\prime}, P_{1}, P_{2}::= & \mathbf{0} & \text { null process } \\
& \operatorname{in}(c, x) \cdot P & \text { input } \\
& \operatorname{out}(c, t) \cdot P & \text { output } \\
& {[s=t] . P} & \text { test }
\end{array}
$$

where $x \in \mathcal{X}, s, t \in \mathcal{T}(\Sigma, \mathcal{N} \cup \mathcal{X})$, and $c \in \mathcal{C} h$.

As usual, a receive action in $(c, x)$ acts as a binding construct for the variable $x$. We assume the usual definitions of free and bound variables for processes. We also assume that each variable is bound at most once. A process is ground if it does not contain any free variables. For sake of conciseness, we sometimes omit the null process at the end of a process.

Following [19], we only consider a minimalistic core calculus that does not include parallel composition. Given that we only consider a bounded number of sessions (i.e., a process calculus without replication) and that we aim at verifying trace equivalence, parallel composition can be added as syntactic sugar to denote the set of all interleavings [19]. Therefore, in this paper, a protocol is simply a finite set of ground processes.

Example 4: Following a description given in [42], we consider the RFID protocol depicted in Figure 1. The reader and the tag $i d$ share the secret key $k$. The reader starts by sending a nonce $r_{1}$. The tag generates a nonce $r_{2}$ and computes the message $t_{0}=\left\langle i d \oplus r_{2}, \mathrm{~h}\left(\left\langle r_{1}, k\right\rangle\right) \oplus r_{2}\right\rangle$ introduced in Example 1]. When receiving such a message, the reader will be able to retrieve $r_{2}$ from the first component, and by xoring it with the second component, he obtains $\mathrm{h}\left(\left\langle r_{1}, k\right\rangle\right)$.

Using our formalism, we can model the two roles of this protocol with the following ground processes:

$$
\begin{aligned}
P_{\text {tag }}= & \operatorname{in}(c, x) . \operatorname{out}\left(c,\left\langle i d \oplus r_{2}, \mathrm{~h}(\langle x, k\rangle) \oplus r_{2}\right\rangle\right) . \mathbf{0} \\
P_{\text {reader }}= & \operatorname{out}\left(c, r_{1}\right) . \operatorname{in}(c, y) . \\
& {\left[\left(\operatorname{proj}_{1}(y) \oplus i d\right) \oplus \operatorname{proj}_{2}(y)=\mathrm{h}\left(\left\langle r_{1}, k\right\rangle\right)\right] . \mathbf{0} }
\end{aligned}
$$

where $r_{1}, r_{2}, k \in \mathcal{N}_{\text {prv }}, i d \in \mathcal{N}_{\text {pub }}$, and $x, y \in \mathcal{X}$. The protocol itself corresponds to the set of ground processes obtained by interleaving these two roles.

The aim of this protocol is not only to authenticate the tag but also to ensure its unlinkability. An attacker should not be able to observe whether he has seen the same tag twice or two different tags. We will formalize this later on, relying on a notion of trace equivalence, and we will show that this protocol fails to achieve this unlinkability property.

To define the semantics of our calculus, we introduce the notion of deducibility. At a particular point in time, after some interaction with a protocol, an attacker may know a sequence of messages $t_{1}, \ldots, t_{\ell} \in \mathcal{T}(\Sigma, \mathcal{N})$. Such a sequence is organised into a frame $\varphi=\left\{w_{1} \mapsto t_{1}, \ldots, w_{\ell} \mapsto t_{\ell}\right\}$ that is a substitution of size $|\varphi|=\ell$.

Definition 3: Let $\varphi$ be a frame, $t \in \mathcal{T}(\Sigma, \mathcal{N})$ and $R \in$ $\mathcal{T}\left(\Sigma, \mathcal{N}_{\text {pub }} \cup \operatorname{dom}(\varphi)\right)$. We say that $t$ is deducible from $\varphi$ using $R$, written $\varphi \vdash_{R} t$, when $R \varphi \downarrow={ }_{\mathrm{AC}} t \downarrow$.

Intuitively, an attacker is able to deduce new messages by applying function symbols in $\Sigma$ to names in $\mathcal{N}_{\text {pub }}$ and terms stored in $\varphi$. The term $R$ is called a recipe.

Example 5: Continuing Example 4, consider the frame

$$
\varphi=\left\{\mathrm{w}_{1} \mapsto r_{1}, \mathrm{w}_{2} \mapsto\left\langle i d \oplus r_{2}, \mathrm{~h}\left(\left\langle r_{1}, k\right\rangle\right) \oplus r_{2}\right\rangle\right\} .
$$

We have that $\mathrm{h}\left(\left\langle r_{1}, k\right\rangle\right)$ is deducible from $\varphi$ using the recipe $R=\left(\operatorname{proj}_{1}\left(\mathrm{w}_{2}\right) \oplus \operatorname{proj}_{2}\left(\mathrm{w}_{2}\right)\right) \oplus i d$.

We now define the semantics of our process calculus by means of a labelled transition relation on configurations. A configuration is a pair $(P, \varphi)$ where $P$ is a ground process, and $\varphi$ is a frame used to record the messages that the participants have sent previously.

The relation $\stackrel{\ell}{\rightarrow}$ where $\ell$ is either an input, an output, or an unobservable action test is defined as follows:

$$
\begin{aligned}
& \operatorname{RECV}(\operatorname{in}(c, x) \cdot P, \varphi) \stackrel{\operatorname{in}(c, R)}{\longrightarrow}(P\{x \mapsto t \downarrow\}, \varphi) \quad \text { if } \varphi \vdash_{R} t \\
& \text { SEND }(\operatorname{out}(c, t) \cdot P, \varphi) \stackrel{\text { out }(c)}{\longrightarrow}\left(P, \varphi \cup\left\{\mathrm{w}_{|\varphi|+1} \mapsto t \downarrow\right\}\right) \\
& \text { TEST }([s=t] . P, \varphi) \stackrel{\text { test }}{\longrightarrow}(P, \varphi) \quad \text { if } s \downarrow=\mathrm{AC} t \downarrow
\end{aligned}
$$

The label in $(c, R)$ indicates the input of a message sent by the attacker over the channel $c$ where $R$ is the recipe that the attacker uses to construct this message. The label out $(c)$ indicates a message sent over channel $c$, and transition rule SEND records the message sent in the frame. Finally, the rule TEST checks equality of $s$ and $t$ in the equational theory and is labelled by the unobservable action test.

Example 6: Consider the ground process $P_{\text {diff }}$ that models an execution of the tag $i d$ (who shares the key $k$ with the reader) followed by an execution of the tag $i d^{\prime}$ (who shares the key $k^{\prime}$ with the reader), i.e., $P_{\text {diff }}=P_{\text {tag }} \cdot P_{\text {tag }}^{\prime}$ where:

$$
\begin{aligned}
& P_{\text {tag }}=\operatorname{in}(c, x) \text {.out }\left(c,\left\langle i d \oplus r_{2}, \mathrm{~h}(\langle x, k\rangle) \oplus r_{2}\right\rangle\right) \\
& P_{\text {tag }}^{\prime}=\operatorname{in}\left(c, x^{\prime}\right) \text {.out }\left(c,\left\langle i d^{\prime} \oplus r_{2}^{\prime}, \mathrm{h}\left(\left\langle x^{\prime}, k^{\prime}\right\rangle\right) \oplus r_{2}^{\prime}\right\rangle\right)
\end{aligned}
$$




$$
\begin{aligned}
& \left(P_{\text {diff }}, \emptyset\right) \stackrel{\operatorname{in}\left(c, r_{1}\right)}{\underset{\operatorname{out}(c)}{\longrightarrow}}\left(\operatorname{out}\left(c,\left\langle i d \oplus r_{2}, \mathrm{~h}\left(\left\langle r_{1}, k\right\rangle\right) \oplus r_{2}\right\rangle\right) \cdot P_{\text {tag }}^{\prime}, \emptyset\right) \\
& \stackrel{\text { out }(c)}{\longrightarrow} \quad\left(P_{\text {tag }}^{\prime},\left\{\mathrm{w}_{1} \mapsto\left\langle i d \oplus r_{2}, \mathrm{~h}\left(\left\langle r_{1}, k\right\rangle\right) \oplus r_{2}\right\rangle\right\}\right) \\
& \stackrel{\operatorname{in}\left(c, r_{1}\right)}{\longrightarrow}\left(\boldsymbol{o u t}\left(c,\left\langle i d^{\prime} \oplus r_{2}^{\prime}, \mathrm{h}\left(\left\langle r_{1}, k^{\prime}\right\rangle\right) \oplus r_{2}^{\prime}\right\rangle\right) . \mathbf{0},\left\{\mathrm{w}_{1} \mapsto\left\langle i d \oplus r_{2}, \mathrm{~h}\left(\left\langle r_{1}, k\right\rangle\right) \oplus r_{2}\right\rangle\right\}\right) \\
& \stackrel{\operatorname{out}(c)}{\longrightarrow} \quad\left(\mathbf{0},\left\{\mathrm{w}_{1} \mapsto\left\langle i d \oplus r_{2}, \mathrm{~h}\left(\left\langle r_{1}, k\right\rangle\right) \oplus r_{2}\right\rangle, \mathrm{w}_{2} \mapsto\left\langle i d^{\prime} \oplus r_{2}^{\prime}, \mathrm{h}\left(\left\langle r_{1}, k^{\prime}\right\rangle\right) \oplus r_{2}^{\prime}\right\rangle\right\}\right) \\
& \left(P_{\text {same }}, \emptyset\right) \stackrel{\operatorname{in}\left(c, r_{1}\right), \operatorname{out}(c), \operatorname{in}\left(c, r_{1}\right), \operatorname{out}(c)}{\longrightarrow}\left(\mathbf{0},\left\{\mathrm{w}_{1} \mapsto\left\langle i d \oplus r_{2}, \mathrm{~h}\left(\left\langle r_{1}, k\right\rangle\right) \oplus r_{2}\right\rangle, \mathrm{w}_{2} \mapsto\left\langle i d \oplus r_{2}^{\prime}, \mathrm{h}\left(\left\langle r_{1}, k\right\rangle\right) \oplus r_{2}^{\prime}\right\rangle\right\}\right)
\end{aligned}
$$

Fig. 2: Some derivations (see Example 6)

We also consider the ground process $P_{\text {same }}$ obtained from $P_{\text {diff }}$ by replacing the occurrence of $i d^{\prime}$ (resp. $k^{\prime}$ ) by $i d$ (resp. $k$ ) (but keeping the nonce $r_{2}^{\prime}$ ). This process models an execution of two instances of the protocol by the same tag $i d$ (who shares the key $k$ with the reader). Following our semantics, we have the derivations described in Figure 2 where $r_{1} \in \mathcal{N}_{\text {pub }}$, i.e. $r_{1}$ is a public name known by the attacker.

When $\ell \neq$ test we define $\stackrel{\ell}{\Rightarrow}$ to be $\stackrel{\text { test }^{*}}{\rightarrow} \stackrel{\ell}{\rightarrow} \stackrel{\text { test }}{\rightarrow}$ and we lift $\stackrel{\ell}{\rightarrow}$ and $\stackrel{\ell}{\Rightarrow}$ to sequences of actions. Given a protocol $\mathcal{P}$, we write $(\mathcal{P}, \varphi) \stackrel{\ell_{1}, \ldots, \ell_{n}}{\longrightarrow}\left(P^{\prime}, \varphi^{\prime}\right)$ if there exists $P \in \mathcal{P}$ such that $(P, \varphi) \stackrel{\ell_{1}, \ldots, \ell_{n}}{\longrightarrow}\left(P^{\prime}, \varphi^{\prime}\right)$, and similarly for $\stackrel{\ell}{\Rightarrow}$.

\section{Process equivalence}

In order to define our notion of equivalence, we first define what it means for a test to hold on a frame.

Definition 4: Let $\varphi$ be a frame and $R_{1}, R_{2}$ be two terms in $\mathcal{T}\left(\Sigma, \mathcal{N}_{\text {pub }} \cup \operatorname{dom}(\varphi)\right)$. The test $R_{1} \stackrel{?}{=} R_{2}$ holds on frame $\varphi$, written $\left(R_{1}=R_{2}\right) \varphi$, if $R_{1} \varphi \downarrow={ }_{\mathrm{AC}} R_{2} \varphi \downarrow$.

Example 7: Consider the frames $\varphi_{\text {diff }}=\left\{\mathrm{w}_{1} \mapsto t, \mathrm{w}_{2} \mapsto t^{\prime}\right\}$ and $\varphi_{\text {same }}=\left\{\mathrm{w}_{1} \mapsto t, \mathrm{w}_{2} \mapsto t^{\prime \prime}\right\}$ where the terms $t, t^{\prime}$, and $t^{\prime \prime}$ are as follows:

- $t=\left\langle i d \oplus r_{2}, \mathrm{~h}\left(\left\langle r_{1}, k\right\rangle\right) \oplus r_{2}\right\rangle$,

- $t^{\prime}=\left\langle i d^{\prime} \oplus r_{2}^{\prime}, \mathrm{h}\left(\left\langle r_{1}, k^{\prime}\right\rangle\right) \oplus r_{2}^{\prime}\right\rangle$,

- $t^{\prime \prime}=\left\langle i d \oplus r_{2}^{\prime}, \mathrm{h}\left(\left\langle r_{1}, k\right\rangle\right) \oplus r_{2}^{\prime}\right\rangle$.

They correspond to the frames obtained at the end of the executions considered in Example 6 The test

$$
\operatorname{proj}_{1}\left(\mathrm{w}_{1}\right) \oplus \operatorname{proj}_{2}\left(\mathrm{w}_{1}\right) \stackrel{?}{=} \operatorname{proj}_{1}\left(\mathrm{w}_{2}\right) \oplus \operatorname{proj}_{2}\left(\mathrm{w}_{2}\right)
$$

holds in $\varphi_{\text {same }}$ but not in $\varphi_{\text {diff. An attacker can xor the }}$ two components of each message and check whether this computation yields an equality or not.

Definition 5: A protocol $\mathcal{P}$ is trace included in a protocol $\mathcal{Q}$, denoted $\mathcal{P} \sqsubseteq \mathcal{Q}$, if whenever $(\mathcal{P}, \emptyset) \stackrel{\ell_{1}, \ldots, \ell_{n}}{\longrightarrow}(P, \varphi)$ and $\left(R_{1}=R_{2}\right) \varphi$, then there exists a configuration $\left(Q^{\prime}, \varphi^{\prime}\right)$ such that $(\mathcal{Q}, \emptyset) \stackrel{\ell_{1}, \ldots, \ell_{n}}{=}\left(Q^{\prime}, \varphi^{\prime}\right)$ and $\left(R_{1}=R_{2}\right) \varphi^{\prime}$. We say that $\mathcal{P}$ and $\mathcal{Q}$ are equivalent, written $\mathcal{P} \approx \mathcal{Q}$, if $\mathcal{P} \sqsubseteq \mathcal{Q}$ and $\mathcal{Q} \sqsubseteq \mathcal{P}$.

This notion of equivalence does not coincide with the usual notion of trace equivalence as defined e.g. in [21]. It is actually coarser and is therefore sound for finding attacks. Moreover, it has been shown that the two notions coincide for the class of determinate processes [19].
Definition 6: We say that a protocol $\mathcal{P}$ is determinate if whenever $(\mathcal{P}, \emptyset) \stackrel{\ell_{1}, \ldots, \ell_{n}}{\longrightarrow}(P, \varphi)$, and $(\mathcal{P}, \emptyset) \stackrel{\ell_{1}, \ldots, \ell_{n}}{\Longrightarrow}\left(P^{\prime}, \varphi^{\prime}\right)$, then for any test $R_{1} \stackrel{?}{=} R_{2}$, we have that: $\left(R_{1}=R_{2}\right) \varphi$ if, and only if $\left(R_{1}=R_{2}\right) \varphi^{\prime}$.

In general, checking determinacy is as difficult as checking equivalence. However, it is typically ensured easily in practice: for instance, any protocol whose roles have a deterministic behaviour can be modeled as a determinate process using a different channel for each role. In case processes are not determinate, the above relation can be used to disprove trace equivalence, i.e., find attacks. It is also possible to check a more fine-grained notion of trace equivalence which implies the usual notion of trace equivalence. This fine-grained notion can be verified straightforwardly by using the algorithm for verifying the above defined (coarse-grained) trace equivalence in a black-box manner, cf. [19] for details.

Example 8: Going back to our running example, we have that $\mathcal{P}_{\text {same }}=\left\{P_{\text {same }}\right\}$ and $\mathcal{P}_{\text {diff }}=\left\{P_{\text {diff }}\right\}$ are not in equivalence according to our definition (as well as the usual notion of trace equivalence since these two protocols are determinate). More precisely, we have that $\mathcal{P}_{\text {same }} \nsubseteq \mathcal{P}_{\text {diff }}$. Indeed, we have shown that:

- $\left(P_{\text {same }}, \emptyset\right) \stackrel{\operatorname{in}\left(c, r_{1}\right), \text { out }(c), \text { in }\left(c, r_{1}\right), \text { out }(c)}{\longrightarrow}\left(\mathbf{0}, \varphi_{\text {same }}\right)$; and - $\left(\operatorname{proj}_{1}\left(\mathrm{w}_{1}\right) \oplus \operatorname{proj}_{2}\left(\mathrm{w}_{1}\right)=\operatorname{proj}_{1}\left(\mathrm{w}_{2}\right) \oplus \operatorname{proj}_{2}\left(\mathrm{w}_{2}\right)\right) \varphi_{\text {same }}$.

However, the only extended trace $\left(P^{\prime}, \varphi^{\prime}\right)$ such that

$$
\left(P_{\text {diff }}, \emptyset\right) \stackrel{\text { in }\left(c, r_{1}\right), \text { out }(c), \text { in }\left(c, r_{1}\right), \text { out }(c)}{\longrightarrow}\left(P^{\prime}, \varphi^{\prime}\right)
$$

is $\left(\mathbf{0}, \varphi_{\text {diff }}\right)$ and we have seen that $\operatorname{proj}_{1}\left(\mathrm{w}_{1}\right) \oplus \operatorname{proj}_{2}\left(\mathrm{w}_{1}\right) \stackrel{?}{=}$ $\operatorname{proj}_{1}\left(w_{2}\right) \oplus \operatorname{proj}_{2}\left(w_{2}\right)$ does not hold in $\varphi_{\text {diff }}$ (see Example 7 .

However, we have that $\mathcal{P}_{\text {diff }} \sqsubseteq \mathcal{P}_{\text {same. }}$. This is a non trivial inclusion that has been checked using our tool.

\section{Modelling USing Horn ClaUses}

Our procedure is based on a fully abstract modelling of a process in first-order Horn clauses.

\section{A. Predicates}

We define symbolic runs, denoted $u, v, w$, as finite sequences of symbolic labels

$$
\ell \in\{\operatorname{in}(c, t), \text { out }(c), \text { test } \mid t \in \mathcal{T}(\Sigma, \mathcal{N} \cup \mathcal{X}), c \in \mathcal{C} h\} .
$$

The empty sequence is denoted $\epsilon$. Intuitively, a symbolic run stands for a set of possible runs of the protocol. We denote $u \sqsubseteq$ AC $v$ when $u$ is a prefix (modulo AC) of $v$. 


$$
\begin{array}{ll}
\left(P_{0}, \varphi_{0}\right) \models \mathrm{r}_{\ell_{1}, \ldots, \ell_{n}} & \text { if }\left(P_{0}, \varphi_{0}\right) \stackrel{L_{1}}{\longrightarrow}\left(P_{1}, \varphi_{1}\right) \ldots \stackrel{L_{n}}{\longrightarrow}\left(P_{n}, \varphi_{n}\right) \text { such that } \ell_{i} \downarrow={ }_{A C} L_{i} \varphi_{i-1 \downarrow} \text { for all } 1 \leq i \leq n \\
\left(P_{0}, \varphi_{0}\right) \models \mathrm{k}_{\ell_{1}, \ldots, \ell_{n}}(R, t) & \text { if when }\left(P_{0}, \varphi_{0}\right) \stackrel{L_{1}}{\longrightarrow}\left(P_{1}, \varphi_{1}\right) \stackrel{L_{2}}{\longrightarrow} \ldots \stackrel{L_{n}}{\longrightarrow}\left(P_{n}, \varphi_{n}\right) \\
& \text { such that } \ell_{i \downarrow}={ }_{\mathrm{AC}} L_{i} \varphi_{i-1 \downarrow} \text { for all } 1 \leq i \leq n, \text { then } \varphi_{n} \vdash_{R} t \\
\left(P_{0}, \varphi_{0}\right) \models \mathrm{i}_{\ell_{1}, \ldots, \ell_{n}}\left(R, R^{\prime}\right) & \text { if there exists } t \text { such that }\left(P_{0}, \varphi_{0}\right) \models \mathrm{k}_{\ell_{1}, \ldots, \ell_{n}}(R, t) \text { and }\left(P_{0}, \varphi_{0}\right) \models \mathrm{k}_{\ell_{1}, \ldots, \ell_{n}}\left(R^{\prime}, t\right) \\
\left(P_{0}, \varphi_{0}\right) \models \operatorname{ri}_{\ell_{1}, \ldots, \ell_{n}}\left(R, R^{\prime}\right) & \text { if }\left(P_{0}, \varphi_{0}\right) \models \mathrm{r}_{\ell_{1}, \ldots, \ell_{n}} \text { and }\left(P_{0}, \varphi_{0}\right) \models \mathrm{i}_{\ell_{1}, \ldots, \ell_{n}}\left(R, R^{\prime}\right)
\end{array}
$$

Fig. 3: Semantics of atomic formulas

We assume a set $\mathcal{Y}$ of recipe variables disjoint from $\mathcal{X}$, and we use capital letters $X, Y, Z$ to range over $\mathcal{Y}$. We assume that such variables may only be substituted by terms in $\mathcal{T}\left(\Sigma, \mathcal{N}_{\text {pub }} \cup \mathcal{W} \cup \mathcal{Y}\right)$. Our logic is based on four predicates, whose semantics is given in Figure 3, where $w$ denotes a symbolic run, $R, R^{\prime}$ are terms in $\mathcal{T}\left(\Sigma, \mathcal{N}_{\text {pub }} \cup \mathcal{W} \cup \mathcal{Y}\right)$, and $t$ is a term in $\mathcal{T}(\Sigma, \mathcal{N} \cup \mathcal{X})$. Intuitively:

- reachability predicate: $r_{w}$ holds when the run represented by $w$ is executable;

- intruder knowledge predicate: $\mathrm{k}_{w}(R, t)$ holds if whenever the run represented by $w$ is executable, the message $t$ can be constructed by the intruder using the recipe $R$;

- identity predicate: $\mathrm{i}_{w}\left(R, R^{\prime}\right)$ holds if whenever the run $w$ is executable, $R$ and $R^{\prime}$ are recipes for the same term; and

- reachable identity predicate: $\operatorname{ri}_{w}\left(R, R^{\prime}\right)$ is a shortcut for $\mathrm{r}_{w} \wedge \mathrm{i}_{w}\left(R, R^{\prime}\right)$.

A (ground) atomic formula is interpreted over a pair consisting of a process $P$ and a frame $\varphi$, and we write $(P, \varphi) \models f$ when the atomic formula $f$ holds for $(P, \varphi)$ or simply $P=f$ when $\varphi$ is the empty frame. We consider first-order formulas built from the above atomic formulas using conjunction, implication and universal quantification. The semantics is as expected, but the domain of quantified variables depends on their type: variables in $\mathcal{X}$ may be mapped to any term in $\mathcal{T}(\Sigma, \mathcal{N})$, while recipe variables in $\mathcal{Y}$ are mapped to recipes, i.e. terms in $\mathcal{T}\left(\Sigma, \mathcal{N}_{\text {pub }} \cup \mathcal{W}\right)$.

Example 9: Continuing our running example, let $w=$ $\operatorname{in}\left(c, r_{1}\right), \operatorname{out}(c), \operatorname{in}\left(c, r_{1}\right), \operatorname{out}(c), t_{0}=i d \oplus \mathrm{h}\left(\left\langle r_{1}, k\right\rangle\right)$, and $R_{i}=\operatorname{proj}_{1}\left(\mathrm{w}_{i}\right) \oplus \operatorname{proj}_{2}\left(\mathrm{w}_{i}\right)$ with $i \in\{1,2\}$. We have that:

$$
\left(P_{\text {same }}, \emptyset\right) \models \mathrm{r}_{w} \wedge \mathrm{k}_{w}\left(R_{1}, t_{0}\right) \wedge \mathrm{k}_{w}\left(R_{2}, t_{0}\right) \wedge \mathrm{ri}_{w}\left(R_{1}, R_{2}\right) .
$$

Consider $t=\left\langle i d \oplus r_{2}, \mathrm{~h}(\langle x, k\rangle) \oplus r_{2}\right\rangle$ and the formulas

- $f_{1}=\forall X, x \cdot \mathrm{r}_{\text {in }(c, x), \text { out }(c)} \Leftarrow \mathrm{k}_{\epsilon}(X, x)$;

- $f_{2}=\forall X, x . \mathrm{k}_{\mathbf{i n}(c, x), \boldsymbol{o u t}(c)}\left(\mathrm{w}_{1}, t\right) \Leftarrow \mathrm{k}_{\epsilon}(X, x)$.

We have that $P_{\text {same }} \models f_{1}$ and $P_{\text {same }} \models f_{2}$.

\section{B. Seed statements}

We shall be mainly concerned with particular forms of Horn clauses which we call statements.

Definition 7: A statement is a Horn clause of the form $H \Leftarrow \mathrm{k}_{u_{1}}\left(X_{1}, t_{1}\right), \ldots, \mathrm{k}_{u_{n}}\left(X_{n}, t_{n}\right)$ where:

- $H \in\left\{\mathrm{r}_{u_{0}}, \mathrm{k}_{u_{0}}(R, t), \mathrm{i}_{u_{0}}\left(R, R^{\prime}\right), \mathrm{ri}_{u_{0}}\left(R, R^{\prime}\right)\right\}$;
- $u_{0}, u_{1}, \ldots, u_{n}$ are symbolic runs such that $u_{i} \sqsubseteq$ AC $u_{0}$ for any $i \in\{1, \ldots, n\}$;

- $t, t_{1}, \ldots, t_{n} \in \mathcal{T}(\Sigma, \mathcal{N} \cup \mathcal{X})$;

- $R, R^{\prime} \in \mathcal{T}\left(\Sigma, \mathcal{N}_{\text {pub }} \cup \mathcal{W} \cup \mathcal{Y}\right)$; and

- $X_{1}, \ldots, X_{n}$ are distinct variables from $\mathcal{Y}$.

Lastly, $\operatorname{vars}(t) \subseteq \operatorname{vars}\left(t_{1}, \ldots, t_{n}\right)$ when $H=\mathrm{k}_{u_{0}}(R, t)$.

In the above definition, we implicitly assume that all variables are universally quantified, i.e., all statements are ground. By abuse of language we sometimes call $\sigma$ a grounding substitution for a statement $H \Leftarrow B_{1}, \ldots, B_{n}$ when $\sigma$ is grounding for each of the atomic formulas $H, B_{1}, \ldots, B_{n}$. The skeleton of a statement $f$, denoted $\operatorname{skel}(f)$, is the statement where recipes are removed.

$$
\begin{aligned}
& \mathrm{r}_{\ell_{1} \sigma \tau \downarrow, \ldots, \ell_{m} \sigma \tau \downarrow} \Leftarrow\left\{\mathrm{k}_{\ell_{1} \sigma \tau \downarrow, \ldots, \ell_{j-1} \sigma \tau \downarrow}\left(X_{j}, x_{j} \sigma \tau \downarrow\right)\right\}_{j \in R(m)} \\
& \text { for all } 0 \leq m \leq n \\
& \text { for all } \sigma \in \operatorname{csu}_{\mathcal{R}, \mathrm{AC}}\left(\left\{s_{k}=t_{k}\right\}_{k \in T(m)}\right) \\
& \text { for all } \tau \in \text { variants }_{\mathcal{R}, \mathrm{AC}}\left(\ell_{1} \sigma, \ldots, \ell_{m} \sigma\right) \\
& \mathrm{k}_{\ell_{1} \sigma \tau \downarrow, \ldots, \ell_{m} \sigma \tau \downarrow}\left(\mathrm{w}_{|S(m)|}, t_{m} \sigma \tau \downarrow\right) \Leftarrow \\
& \text { for all } m \in S(n) \\
& \left\{\mathrm{k}_{\ell_{1} \sigma \tau \downarrow, \ldots, \ell_{j-1} \sigma \tau \downarrow}\left(X_{j}, x_{j} \sigma \tau \downarrow\right)\right\}_{j \in R(m)} \\
& \text { for all } \sigma \in \operatorname{csu}_{\mathcal{R}, \mathrm{AC}}\left(\left\{s_{k}=t_{k}\right\}_{k \in T(m)}\right) \\
& \text { for all } \tau \in \text { variants }_{\mathcal{R}, \mathrm{AC}}\left(\ell_{1} \sigma, \ldots, \ell_{m} \sigma, t_{m} \sigma\right) \\
& \mathrm{k}_{\epsilon}(c, c) \Leftarrow \\
& \text { for all public names } c \in \mathcal{N}_{\text {pub }}^{0} \\
& \mathrm{k}_{\ell_{1}, \ldots, \ell_{m}}\left(\mathrm{f}\left(Y_{1}, \ldots, Y_{k}\right), \mathrm{f}\left(y_{1}, \ldots, y_{k}\right) \tau \downarrow\right) \Leftarrow \\
& \left\{\mathrm{k}_{\ell_{1}, \ldots, \ell_{m}}\left(Y_{j}, y_{j} \tau \downarrow\right)\right\}_{j \in\{1, \ldots, k\}}
\end{aligned}
$$

Fig. 4: Seed statements

As mentioned earlier, our decision procedure is based on a fully abstract modelling of a process in first-order Horn clauses. In this section, given a ground process $P$ we will give a set of statements seed $(P)$ which will serve as a starting point for the modelling. We shall also establish that the set of statements $\operatorname{seed}(P)$ is a sound and (partially) complete abstraction of the ground process $P$. In order to formally define seed $(P)$, we start by fixing some conventions. 


$$
\begin{array}{cc}
f_{0}^{+}: & \mathrm{k}_{w}\left(X_{1} \oplus X_{2}, x_{1} \oplus x_{2}\right) \Leftarrow \mathrm{k}_{w}\left(X_{1}, x_{1}\right), \mathrm{k}_{w}\left(X_{2}, x_{2}\right) \\
f_{1}^{+}: & \mathrm{k}_{w}\left(X_{1} \oplus X_{2}, x_{1}\right) \Leftarrow \mathrm{k}_{w}\left(X_{1}, x_{1} \oplus x_{2}\right), \mathrm{k}_{w}\left(X_{2}, x_{2}\right) \\
f_{2}^{+}: & \mathrm{k}_{w}\left(X_{1} \oplus X_{2}, x_{1} \oplus x_{2}\right) \Leftarrow \\
& \mathrm{k}_{w}\left(X_{1}, x_{1} \oplus x_{3}\right), \mathrm{k}_{w}\left(X_{2}, x_{2} \oplus x_{3}\right) \\
f_{3}^{+}: & \mathrm{k}_{w}\left(X_{1} \oplus X_{2}, 0\right) \Leftarrow \mathrm{k}_{w}\left(X_{1}, x\right), \mathrm{k}_{w}\left(X_{2}, x\right) \\
f_{4}^{+}: & \mathrm{k}_{w}\left(X_{1} \oplus X_{2}, x\right) \Leftarrow \mathrm{k}_{w}\left(X_{1}, x\right), \mathrm{k}_{w}\left(X_{2}, 0\right)
\end{array}
$$

where $w=\ell_{1}, \ldots, \ell_{m}$ is as defined in Section III-B.

Fig. 5: Definition of $f_{0}^{+}$and its variants.

Let $P=\mathrm{a}_{1} \cdot \mathrm{a}_{2} \ldots . \mathrm{a}_{n}$ be a ground process. We assume w.l.o.g. the following naming conventions:

1) if $\mathrm{a}_{i}$ is a receive action then $\mathrm{a}_{i}=\ell_{i}=\mathbf{i n}\left(c_{i}, x_{i}\right)$;

2) if $\mathrm{a}_{i}$ is a send action then $\mathrm{a}_{i}=\operatorname{out}\left(c_{i}, t_{i}\right), \ell_{i}=\operatorname{out}\left(c_{i}\right)$;

3) if $\mathrm{a}_{i}$ is a test actions then $\mathrm{a}_{i}=\left[s_{i}=t_{i}\right]$, and $\ell_{i}=$ test.

Moreover, we assume that $x_{i} \neq x_{j}$ for any $i \neq j$.

For each $0 \leq m \leq n$, let the sets $R(m), S(m)$ and $T(m)$ respectively denote the set of indices of the receive, send and test actions amongst $\mathrm{a}_{1}, \ldots, \mathrm{a}_{m}$. Moreover, we denote by $|S|$ the number of elements in such a set. Formally,

- $R(m)=\left\{i \mid 1 \leq i \leq m\right.$ and $\left.\mathbf{a}_{i}=\mathbf{i n}\left(c_{i}, x_{i}\right)\right\}$;

- $S(m)=\left\{i \mid 1 \leq i \leq m\right.$ and $\left.\mathrm{a}_{i}=\operatorname{out}\left(c_{i}, t_{i}\right)\right\}$;

- $T(m)=\left\{i \mid 1 \leq i \leq m\right.$ and $\left.\mathrm{a}_{i}=\left[s_{i}=t_{i}\right]\right\}$.

Given a set of public names $\mathcal{N}_{\text {pub }}^{0} \subseteq \mathcal{N}_{\text {pub }}$, the set of seed statements associated to $P$ and $\mathcal{N}_{\text {pub }}^{0}$, denoted $\operatorname{seed}\left(P, \mathcal{N}_{\text {pub }}^{0}\right)$, is defined in Figure 4. We may note that while constructing the set of seed statements, we compute a complete set of unifiers modulo the whole equational theory $\mathrm{E}$ w.r.t. all tests. In addition, we also apply finite variants. This allows us to get rid of the rewriting theory in the remainder of our procedure.

The first kind of seed statement models the fact that the run represented by $\ell_{1} \sigma \tau \downarrow, \ldots, \ell_{m} \sigma \tau \downarrow$ is executable as soon as the attacker is able to feed each input with terms that will allow one to successfully pass all the tests. Following the same idea, under the same hypotheses, the attacker will be able to learn the output term. The two last families of statements model the deduction capabilities of the attacker who knows all the public names, and is able to apply a function symbol on top of terms that he already knows. These abilities can be used at any stage. However, since we will give the attacker the abilities to transfer his knowledge, we only have to express the fact that he knows public names initially.

If $\mathcal{N}_{\text {pub }}^{0}=\mathcal{N}_{\text {pub }}$, then $\operatorname{seed}\left(P, \mathcal{N}_{\text {pub }}\right)$ is said to be the set of seed statements associated to $P$ and in this case we write $\operatorname{seed}(P)$ as a $\operatorname{shortcut}$ for $\operatorname{seed}\left(P, \mathcal{N}_{\text {pub }}\right)$.

Example 10: Continuing our running example, let

- $u=\operatorname{in}(c, x)$.out $(c), v=\operatorname{in}\left(c, x^{\prime}\right)$.out $(c)$;

- $t=\left\langle i d \oplus r_{2}, \mathrm{~h}(\langle x, k\rangle) \oplus r_{2}\right\rangle$;

- $t^{\prime}=\left\langle i d \oplus r_{2}, \mathrm{~h}\left(\left\langle x^{\prime}, k\right\rangle\right) \oplus r_{2}\right\rangle$.

The following statements belong to seed $\left(P_{\text {same }}\right)$ :

$$
\begin{array}{rlrl}
\mathrm{r}_{u} & \Leftarrow \mathrm{k}_{\epsilon}(X, x) & \mathrm{r}_{u v} & \Leftarrow \mathrm{k}_{w}\left(X^{\prime}, x^{\prime}\right), \mathrm{k}_{\epsilon}(X, x) \\
\mathrm{k}_{u}\left(\mathrm{w}_{1}, t\right) & \Leftarrow \mathrm{k}_{\epsilon}(X, x) \quad \mathrm{k}_{u v}\left(\mathrm{w}_{2}, t^{\prime}\right) & \Leftarrow \mathrm{k}_{w}\left(X^{\prime}, x^{\prime}\right), \mathrm{k}_{\epsilon}(X, x)
\end{array}
$$

When considering the last kind of statements in Figure 4 with $f=\operatorname{proj}_{1}$ and the empty run, we obtain:

$$
\begin{aligned}
\mathrm{k}_{\epsilon}\left(\operatorname{proj}_{1}(X), \operatorname{proj}_{1}(x)\right) & \Leftarrow \mathrm{k}_{\epsilon}(X, x) \\
\mathrm{k}_{\epsilon}\left(\operatorname{proj}_{1}(X), x_{1}\right) & \Leftarrow \mathrm{k}_{\epsilon}\left(X,\left\langle x_{1}, x_{2}\right\rangle\right)
\end{aligned}
$$

Figure 5 shows those obtained for $\mathrm{f}=\oplus$ and an arbitrary $w$.

\section{Soundness and completeness}

We show that as far as reachability and intruder knowledge predicates are concerned, the set $\operatorname{seed}(P)$ is a complete abstraction of a process. However, we need one more definition to state this fact.

Definition 8: Given a set $K$ of statements, $\mathcal{H}(K)$ is the smallest set of ground facts such that:

$$
f=\left(H \Leftarrow B_{1}, \ldots, B_{n}\right) \in K
$$

$\sigma$ grounding for $f$ with skel $(f \sigma)$ in normal form

ConseQ $\frac{B_{1} \sigma \in \mathcal{H}(K), \ldots, B_{n} \sigma \in \mathcal{H}(K)}{H \sigma \in \mathcal{H}(K)}$

$\operatorname{EXTEND} \frac{\mathrm{k}_{u}(R, t) \in \mathcal{H}(K)}{\mathrm{k}_{u v}(R, t) \in \mathcal{H}(K)}$

Theorem 1: Let $P$ be a ground process.

- $P \models f$ for any $f \in \operatorname{seed}(P) \cup \mathcal{H}(\operatorname{seed}(P))$;

- If $(P, \emptyset) \stackrel{L_{1}, \ldots, L_{m}}{\longrightarrow}(Q, \varphi)$ for some $(Q, \varphi)$, then

1) $r_{L_{1} \varphi \downarrow, \ldots, L_{m} \varphi \downarrow} \in \mathcal{H}(\operatorname{seed}(P))$; and

2) if $\varphi \vdash_{R} t$ then $\mathrm{k}_{L_{1} \varphi \downarrow, \ldots, L_{m} \varphi \downarrow}(R, t \downarrow) \in \mathcal{H}(\operatorname{seed}(P))$.

We will see that the completeness of $\operatorname{seed}(P)$ can be built upon to achieve full abstraction, i.e., also including identities of the process $P$ and a procedure for checking equivalence.

\section{SATURATION}

We shall now describe how to verify equivalence given the protocol representation as Horn clauses introduced in the previous section. Given a ground process $P$ and a protocol $\mathcal{Q}$, we saturate the set of seed statements for $P$ to construct a set of simple statements which we will call solved statements. The saturation procedure ensures that the set of solved statements is a complete abstraction of $P$. Then, we use the resulting solved statements to decide whether $P$ is trace included in $\mathcal{Q}$. Repeating this procedure for all $P \in \mathcal{P}$, and doing similarly for processes in $\mathcal{Q}$, we are then able to decide whether two determinate protocols $\mathcal{P}$ and $\mathcal{Q}$ are in trace equivalence.

In this section we will describe the saturation procedure. It manipulates a set of statements called a knowledge base.

Definition 9: Given a statement $f=\left(H \Leftarrow B_{1}, \ldots, B_{n}\right)$,

- $f$ is said to be solved if for all $1 \leq i \leq n$, we have that $B_{i}=\mathrm{k}_{\ell_{1}, \ldots, \ell_{j_{i}}}\left(X_{i}, x_{i}\right)$ for some $x_{i} \in \mathcal{X}$, and $X_{i} \in \mathcal{Y}$.

- $f$ is said to be well-formed if whenever it is solved and $H=\mathrm{k}_{\ell_{1}, \ldots, \ell_{k}}(R, t)$, we have that $t \notin \mathcal{X}$.

A set of well-formed statements is called a knowledge base. If $K$ is a knowledge base, $K_{\text {solved }}=\{f \in K \mid f$ is solved $\}$. 
Given an initial knowledge base $K$, the saturation procedure is a non-deterministic process which produces another knowledge base. At each step of the saturation procedure, a new statement is generated and the knowledge base is updated with the new statement. This two-step process is repeated until a fixed point is reached. We denote by sat $(K)$ the set of reachable fixed points starting from the initial set $K$.

Before describing these two steps in Section IV-B, we explain in the following section why a naive adaptation of the original AKISS procedure would not be effective.

\section{A. Difficulties}

In the original procedure [19], the saturated knowledge base is obtained by applying (among others) the following resolution rule based on most general unifiers (mgu):

$$
\begin{gathered}
f=\left(H \Leftarrow \mathrm{k}_{u v}(X, t), B_{1}, \ldots, B_{n}\right) \in K \\
g=\left(\mathrm{k}_{w}\left(R, t^{\prime}\right) \Leftarrow B_{n+1}, \ldots, B_{m}\right) \in K_{\text {solved }} \\
\sigma=\operatorname{mgu}\left(\mathrm{k}_{u}(X, t), \mathrm{k}_{w}\left(R, t^{\prime}\right)\right) \quad t \notin \mathcal{X} \\
K=K \cup h
\end{gathered}
$$

where $h=\left(\left(H \Leftarrow B_{1}, \ldots, B_{n}, B_{n+1}, \ldots, B_{m}\right) \sigma\right)$.

This rule is close to a standard resolution step, between an unsolved statement $f$ and a solved deduction statement $g$. Note, however, that we do not impose the two symbolic runs involved in this resolution step to be unifiable but we only require that $w$ is unifiable with a prefix of the other symbolic run (namely $u$ ). Intuitively, this comes from the fact that the knowledge of the attacker is monotone (the attacker never forgets any data), and a term $t^{\prime} \sigma$ deducible in the run $w \sigma$ will remain deducible in any extension of this run.

For the sake of simplicity, we consider here that the update operator $U$ simply adds $h$ to $K$. A naive approach to add the xor operator consists in replacing the condition $\sigma=$ $\operatorname{mgu}\left(\mathrm{k}_{u}(X, t), \mathrm{k}_{w}\left(R, t^{\prime}\right)\right)$ by $\sigma \in \operatorname{csu}_{\mathrm{AC}}\left(\mathrm{k}_{u}(X, t), \mathrm{k}_{w}\left(R, t^{\prime}\right)\right)$, i.e, performing unification modulo $A C$ instead of simply computing the mgu between these two terms. The obtained procedure would be correct but would rarely terminate.

Example 11: Let $P=\operatorname{in}\left(c, z_{1}\right) \cdot \operatorname{in}\left(c, z_{2}\right) \cdot\left[z_{2}=a \oplus z_{1}\right] .0$ and $w=\operatorname{in}\left(c, z_{1}\right)$, in $\left(c, a \oplus z_{1}\right)$. The set $\operatorname{seed}(P)$ contains (among others) the following statements:

$$
\begin{aligned}
\mathrm{r}_{w, \text { test }} & \Leftarrow \mathrm{k}_{\epsilon}\left(Z_{1}, z_{1}\right), \mathrm{k}_{w}\left(Z_{2}, a \oplus z_{1}\right) \\
\mathrm{k}_{w}(X \oplus Y, x \oplus y) & \Leftarrow \mathrm{k}_{w}(X, x), \mathrm{k}_{w}(Y, y)
\end{aligned}
$$

The resolution rule can be applied on these two statements, and one of the substitutions is

$$
\sigma=\left\{x \mapsto a \oplus z_{11}, y \mapsto z_{12}, z_{1} \mapsto z_{11} \oplus z_{12}\right\}
$$

resulting in the following statement:

$$
\mathrm{r}_{w \sigma} \Leftarrow \mathrm{k}_{\epsilon}\left(Z_{1}, z_{11} \oplus z_{12}\right), \mathrm{k}_{w \sigma}\left(X, a \oplus z_{11}\right), \mathrm{k}_{w \sigma}\left(Y, z_{12}\right) .
$$

This statement can again be resolved using the same statement as before, yielding an infinite loop.

\section{B. Saturation procedure}

We now explain our saturation procedure which is inspired from the one given in [19]. First, as expected, we perform resolution modulo associativity and commutativity (AC) to capture algebraic properties of xor. Second, in order to achieve termination in practice, we constrained the resolution rules in various ways while preserving completeness of our procedure.

1) Generating new statements: Given a knowledge base $K$, new statements $f$ are generated by applying the rules in Figure 6 Each of these rules generates a new statement $h$. Roughly, as already explained, the rule RESOLUTION applies the standard rule of resolution from first-order logic between an unsolved statement $f$ and a solved deduction statement $g$ and allows us to propagate constraints imposed from a partial execution of a trace to its possible extensions through the unification of the symbolic runs $u$ and $w$.

The rule RESOLUTION+ does essentially the same for the statement $g=f_{0}^{+}$but applies some special treatments to avoid non-termination issues. The rule EQUATION allows us to derive new identities on recipes that may be imposed by the execution of the protocol. The rule TEST allows us to conclude which identities necessarily hold in an execution of the protocol. Once the statement $h$ is generated, we update the knowledge base $K$ with $h$. This process is explained below.

More precisely, we restrict the use of the resolution rule and we only apply it on a selected atom. To formalise this, we assume a selection function sel which returns $\perp$ when applied on a solved statement, and an atom $\mathrm{k}_{w}(X, t)$ with $t \notin \mathcal{X}$ when applied on an unsolved statement. Resolution must be performed on this selected atom.

In order to avoid the termination problem illustrated in the previous section when considering equational theories that include xor, we introduce a marking on atomic formulas in the hypothesis of unsolved statements. The marking is used to disallow resolution against a statement in $f_{0}^{+}$. We denote unsolved statements with their marking as

$$
H \Leftarrow B_{1}, \ldots B_{n} \| \mathcal{M}
$$

where $\mathcal{M} \subseteq\left\{B_{1}, \ldots, B_{n}\right\}$ is the set of hypotheses of the statement which are marked. Marking will only be used for unsolved statements, and we implicitly set an empty marking on newly generated solved statements. Statements in $f_{1}^{+}$ and $f_{2}^{+}$will be marked directly when constructing the initial knowledge base. More precisely, we mark the two hypotheses of any statement in $f_{1}^{+} \cup f_{2}^{+}$(see Definition 14. Intuitively, completeness is preserved as derivation trees in $\mathcal{H}(K)$ can always be reorganised by pushing the use of CONSEQ rules with statement in $f_{0}^{+}$below those with statements in $f_{1}^{+}$ and $f_{2}^{+}$. Other statements will be marked dynamically in rule RESOLUTION+, i.e., when performing resolution against a statement in $f_{0}^{+}$: to decide which of the two new hypotheses has to be marked we rely on the following notions.

Definition 10: Given a term $t$, we define factor $(t)=$ $\left\{t_{1}, \ldots, t_{n}\right\}$ when $\bigoplus_{i} t_{i}=t$ and none of the $t_{i}$ is itself a 
sum. The function $\operatorname{rigid}(t)$ returns a term $t_{i} \in$ factor $(t)$ such that $t_{i} \notin \mathcal{X}$ or $\perp$ if no such $t_{i}$ exists.

When performing RESOLUTION+ with a selected atom for which a rigid factor can be found, we mark the hypothesis of the generated statements that contains the factor returned by rigid. This factor has to be rigid in the sense that it cannot be a variable. Again, we can show that we preserve completeness when marking this hypothesis. When we need to derive a term which is a sum, and we decide to split this sum in two parts, we will assume that the chosen rigid factor has to be obtained in an atomic way (it cannot be the result of a sum anymore). This amounts to favour one arrangement among all the possible ones up to associativity and commutativity of the xor operator.

Example 12: Going back to Example 11, we have that RESOLUTION+ will be applied between these two statements, and $\operatorname{rigid}\left(a \oplus z_{1}\right)$ necessarily returns $a$. Therefore, the resulting statement becomes:

$$
\mathrm{r}_{w \sigma} \Leftarrow \mathrm{k}_{\epsilon}\left(Z_{1}, z_{11} \oplus z_{12}\right), \mathrm{k}_{w \sigma}\left(X, a \oplus z_{11}\right), \mathrm{k}_{w \sigma}\left(Y, z_{12}\right) \| \mathcal{M}
$$

where $\mathcal{M}=\left\{\mathrm{k}_{w \sigma}\left(X, a \oplus z_{11}\right)\right\}$.

This forbids the use of RESOLUTION+ on $\mathrm{k}_{w \sigma}\left(X, a \oplus z_{11}\right)$. Provided that our function sel returns $\mathrm{k}_{w \sigma}\left(X, a \oplus z_{11}\right)$, the saturation procedure can now only do a RESOLUTION rule on the next statement and therefore the non termination issue mentioned in Example 11 is avoided.

Example 13: The marking of $f_{1}^{+}$and $f_{2}^{+}$is also important to ensure termination in practice. Indeed, otherwise, it would be possible to apply the RESOLUTION+ rule between $f_{1}^{+}$(and $f_{0}^{+}$) on the atom $\mathrm{k}_{w}\left(X_{1}, x_{1} \oplus x_{2}\right)$. However, the term $x_{1} \oplus x_{2}$ has no rigid factor, and among the resulting statements, we will find the following one:

$$
\begin{aligned}
\mathrm{k}_{w \sigma}\left(X_{1} \oplus X_{2} \oplus X_{3}, x_{11} \oplus x_{12}\right) \Leftarrow & \mathrm{k}_{w \sigma}\left(X_{1}, x_{11} \oplus x_{21}\right), \\
& \mathrm{k}_{w \sigma}\left(X_{2}, x_{12} \oplus x_{22}\right), \\
& \mathrm{k}_{w \sigma}\left(X_{3}, x_{21} \oplus x_{22}\right) \| \emptyset
\end{aligned}
$$

As no literal is marked, whatever is the selection function, the Resolution+ rule could be applied and the saturation procedure would enter an infinite loop.

Finally, the RESOLUTION and RESOLUTION+ rules induce a parent/child relationship between the unsolved statement used in the rule and the generated statement, which will eventually be added to the knowledge base after canonization (see below). This relation allows us to define the ancestor of any statement to be the parent of its parent etc. until we reach an unsolved statement in the initial knowledge base. In the following, we need to distinguish all deduction statements whose oldest ancestor belongs to $f_{1}^{+} \cup f_{2}^{+}$(with marking). We call these statements VIP statement, and they will deserve a privileged treatment in the update.

2) Update: We will now define the update operator 4 which adds statements generated by the rules of Figure 6 to the knowledge base. We first need to introduce the set of consequences of a knowledge base.
Definition 11: Let $K$ be a knowledge base, the set of consequences, conseq $(K)$, is the smallest set such that

$$
\begin{aligned}
& \text { AXIOM } \overline{\mathrm{k}_{u v}(R, t) \Leftarrow \mathrm{k}_{u}(R, t), B_{1}, \ldots, B_{m} \in \operatorname{conseq}(K)} \\
& \mathrm{k}_{u}(R, t) \Leftarrow B_{1}, \ldots, B_{n} \in K \quad \sigma \text { a substitution } \\
& \operatorname{RES} \frac{B_{i} \sigma \Leftarrow C_{1}, \ldots, C_{m} \in \operatorname{conseq}(K) 1 \leq i \leq n}{\mathrm{k}_{u v}(R, t) \sigma \Leftarrow C_{1}, \ldots, C_{m} \in \operatorname{conseq}(K)}
\end{aligned}
$$

We shall see that a weak form of update is sufficient when considering a deduction statement that is already a consequence of the knowledge base up to a change of recipe.

Definition 12: The canonical form $f \Downarrow$ of a statement

$$
f=\left(H \Leftarrow B_{1}, \ldots, B_{n} \| \mathcal{M}\right)
$$

is the statement obtained by first normalizing all the recipes, then applying the rule REMOVE as many times as possible, and for solved deduction statement, applying the rule SHIFT as many times as possible.

$$
\begin{gathered}
H \Leftarrow \mathrm{k}_{u v}(X, t), \mathrm{k}_{u}(Y, t), B_{1}, \ldots, B_{n} \| \mathcal{M} \\
\operatorname{Remove} \frac{\text { with } X \notin \operatorname{vars}(H)}{H \Leftarrow \mathrm{k}_{u}(Y, t), B_{1}, \ldots, B_{n} \| \mathcal{M} \backslash \mathrm{k}_{u v}(X, t)}
\end{gathered}
$$

SHIFT $\frac{\mathrm{k}_{u v}(R, t) \Leftarrow \mathrm{k}_{u}(X, x), B_{1}, \ldots, B_{n} \text { with } x \in \text { factor }(t)}{\mathrm{k}_{u v}(R \oplus X, t \oplus x \downarrow) \Leftarrow \mathrm{k}_{u}(X, x), B_{1}, \ldots, B_{n}}$

Definition 13: Let $K$ be a knowledge base, and $f$ a statement. The update of $K$ by $f$, denoted $K \uplus f$, is $K$ when skel $(f)$ is not in normal form (the statement is dropped). Otherwise, two options are possible:

- $K \cup f=K \cup\{f \Downarrow\}$;

- $K \uplus f=K \cup\left\{\mathrm{i}_{w}\left(R \downarrow, R_{\downarrow}^{\prime} \downarrow\right) \Leftarrow B_{1}, \ldots B_{n}\right\}$ provided that

- $f \Downarrow=\left(\mathrm{k}_{w}(R, t) \Leftarrow B_{1}, \ldots B_{n}\right)$, and

- $f$ is a solved statement but not a VIP one, and

- $\left(\mathrm{k}_{w}\left(R^{\prime}, t\right) \Leftarrow B_{1}, \ldots B_{n}\right) \in \operatorname{conseq}\left(K_{\text {solved }}\right)$.

Note that the update is not a function because there may be several $R^{\prime}$ for which the second option can be chosen. Even when such an $R^{\prime}$ exists, we may still update the base by choosing the first option. These choices are implementation details, and our results hold regardless.

3) Initial knowledge base: We finally define on which knowledge base we initiate the saturation procedure.

Definition 14: Let $P$ be a ground process, and $\mathcal{N}_{\text {pub }}^{0}$ be a set of names. We have that

$$
\operatorname{seed}\left(P, \mathcal{N}_{\text {pub }}^{0}\right)=f_{0}^{+} \uplus f_{1}^{+} \uplus f_{2}^{+} \uplus f_{3}^{+} \uplus f_{4}^{+} \uplus S
$$

where $f_{i}$ (with $0 \leq i \leq 4$ ) are defined as given in Figure 5 . and $S$ are the remaining statements. Let $K_{0}$ be the set of statements which contains:

1) deduction statements in $f_{0}^{+} \cap \operatorname{seed}\left(P, \mathcal{N}_{\text {pub }}^{0}\right)$; 


$$
\begin{aligned}
& f=\left(H \Leftarrow \mathrm{k}_{u v}(X, t), B_{1}, \ldots, B_{n} \| \mathcal{M}\right) \in K \text { such that } \mathrm{k}_{u v}(X, t)=\operatorname{sel}(f) \\
& \text { RESOLUtion } \frac{g=\left(\mathrm{k}_{w}\left(R, t^{\prime}\right) \Leftarrow B_{n+1}, \ldots, B_{m}\right) \in K_{\text {solved }} \backslash f_{0}^{+}}{K=K \uplus\left\{h \sigma \mid \sigma \in \operatorname{csu}_{\mathrm{AC}}\left(\mathrm{k}_{u}(X, t), \mathrm{k}_{w}\left(R, t^{\prime}\right)\right)\right\}} \\
& \text { where } h=\left(H \Leftarrow B_{1}, \ldots, B_{n}, B_{n+1}, \ldots, B_{m} \| \mathcal{M} \backslash\left\{\mathrm{k}_{u v}(X, t)\right\}\right) \\
& \text { Resolution }+\frac{f=\left(H \Leftarrow \mathrm{k}_{u}(X, t), B_{1}, \ldots, B_{n} \| \mathcal{M}\right) \in K \text { such that } \mathrm{k}_{u}(X, t)=\operatorname{sel}(f) \text { and } \mathrm{k}_{u}(X, t) \notin \mathcal{M}}{K=K \uplus\left\{h \sigma \mid \sigma \in \operatorname{csu}_{\mathrm{AC}}\left(\langle X, t\rangle,\left\langle X_{1} \oplus X_{2}, x_{1} \oplus x_{2}\right\rangle\right)\right\}} \\
& \text { where } h=\left(H \Leftarrow B_{1}, \ldots, B_{n}, \mathrm{k}_{u}\left(X_{1}, x_{1}\right), \mathrm{k}_{u}\left(X_{2}, x_{2}\right) \| \mathcal{M}^{\prime}\right) \\
& \text { and } \mathcal{M}^{\prime}=\mathcal{M} \cup\left\{\mathrm{k}_{u}\left(X_{i}, x_{i}\right) \mid \operatorname{rigid}(t) \sigma \in \text { factor }\left(x_{i} \sigma\right) \text { and } i \in\{1,2\}\right\} \\
& \text { EQUATION } \frac{f, g \in\left(K_{\text {solved }} \backslash f_{0}^{+}\right) \quad f=\left(\mathrm{k}_{u}(R, t) \Leftarrow B_{1}, \ldots, B_{n}\right) \quad g=\left(\mathrm{k}_{u^{\prime} v^{\prime}}\left(R^{\prime}, t^{\prime}\right) \Leftarrow B_{n+1}, \ldots, B_{m}\right)}{K=K \uplus\left\{h \sigma \mid \sigma \in \operatorname{csu}_{\mathrm{AC}}\left(\langle u, t\rangle,\left\langle u^{\prime}, t^{\prime}\right\rangle\right)\right\} \text { where } h=\left(\mathrm{i}_{u^{\prime} v^{\prime}}\left(R, R^{\prime}\right) \Leftarrow B_{1}, \ldots, B_{m}\right)} \\
& \text { TEST } \frac{f, g \in K_{\text {solved }}, \quad f=\left(\mathrm{i}_{u}\left(R, R^{\prime}\right) \Leftarrow B_{1}, \ldots, B_{n}\right) \quad g=\left(\mathrm{r}_{u^{\prime} v^{\prime}} \Leftarrow B_{n+1}, \ldots, B_{m}\right)}{K=K \uplus\left\{h \sigma \mid \sigma \in \operatorname{csu}_{\mathrm{AC}}\left(u, u^{\prime}\right)\right\} \text { where } h=\left(\mathrm{ri}_{u^{\prime} v^{\prime}}\left(R, R^{\prime}\right) \Leftarrow B_{1}, \ldots, B_{m}\right)}
\end{aligned}
$$

Fig. 6: Saturation rules

2) deduction statements in $\left(f_{1}^{+} \cup f_{2}^{+}\right) \cap \operatorname{seed}\left(P, \mathcal{N}_{\text {pub }}^{0}\right)$ with their two hypotheses marked:

$$
\mathrm{k}_{w}\left(X_{1} \oplus X_{2}, t\right) \Leftarrow B_{1}, B_{2} \|\left\{B_{1}, B_{2}\right\} .
$$

The initial knowledge base associated to $\operatorname{seed}\left(P, \mathcal{N}_{\text {pub }}^{0}\right)$, denoted $K_{\text {init }}\left(\operatorname{seed}\left(P, \mathcal{N}_{\text {pub }}^{0}\right)\right)$, is defined to be $K_{0}$ updated by the set $S$, i.e.,

$$
K_{\text {init }}\left(\operatorname{seed}\left(P, \mathcal{N}_{\text {pub }}^{0}\right)\right)=\left(\left(\left(K_{0} \uplus g_{1}\right) \uplus g_{2}\right) \ldots g_{k}\right)
$$

where $g_{1}, \ldots, g_{k}$ is an enumeration of the statements in $S$. We sometimes write $K_{\text {init }}(P)$ for $K_{\text {init }}\left(\operatorname{seed}\left(P, \mathcal{N}_{\text {pub }}^{0}\right)\right)$.

When building the initial knowledge base we first add some of the variants related to $\oplus$. Note in particular that we mark all hypotheses of the variants in $f_{1}^{+}$and $f_{2}^{+}$(Figure 5), and we do not add statements in $f_{3}^{+}$and $f_{4}^{+}$. All other seed statements are simply added using the update operator.

Please observe that $K_{\text {init }}(P)$ depends on the order in which statements in $\operatorname{seed}(P)$ are updated. The exact order, however, is not important and our results shall hold regardless of the chosen order. The saturation procedure takes $K_{\text {init }}(P)$ as an input and produces a knowledge base $K_{\text {sat }} \in \operatorname{sat}\left(K_{\text {init }}(P)\right)$. The reason for choosing $K_{\text {init }}(P)$ instead of seed $(P)$ as the starting point of the saturation procedure is that $\operatorname{seed}(P)$ may not be a knowledge base (recall that a knowledge base is a set of well-formed statements). The fact that the set $K_{\text {init }}(P)$ is, however, a knowledge base follows directly from the fact that we apply our SHIFT rule (through the canonization process) before adding a deduction statement to the current set.

$$
\begin{gathered}
\operatorname{REFL} \frac{\mathrm{i}_{w}(R, R) \in \mathcal{H}_{\mathrm{e}}(K)}{\operatorname{ExT} \frac{\mathrm{i}_{u}\left(R, R^{\prime}\right) \in \mathcal{H}_{\mathrm{e}}(K)}{\mathrm{i}_{u v}\left(R, R^{\prime}\right) \in \mathcal{H}_{\mathrm{e}}(K)}} \\
\operatorname{CONG} \frac{\mathrm{i}_{w}\left(R_{1}, R_{1}^{\prime}\right), \ldots, \mathrm{i}_{w}\left(R_{n}, R_{n}^{\prime}\right) \in \mathcal{H}_{\mathrm{e}}(K) \mathrm{f} \in \Sigma}{\mathrm{i}_{w}\left(\mathrm{f}\left(R_{1}, \ldots R_{n}\right), \mathrm{f}\left(R_{1}^{\prime}, \ldots R_{n}^{\prime}\right)\right) \in \mathcal{H}_{\mathrm{e}}(K)} \\
\operatorname{MOD-I} \frac{\mathrm{i}_{w}\left(R_{1}, R_{2}\right) \in \mathcal{H}_{\mathrm{e}}(K) R_{i \downarrow} \downarrow{ }_{\mathrm{AC}} R_{i \downarrow}^{\prime} \downarrow i \in\{1,2\}}{\mathrm{i}_{w}\left(R_{1}^{\prime}, R_{2}^{\prime}\right) \in \mathcal{H}_{\mathrm{e}}(K)} \\
\operatorname{MOD-RI} \frac{\mathrm{ri}_{w}\left(R_{1}, R_{2}\right) \in \mathcal{H}_{\mathrm{e}}(K) R_{i} \downarrow={ }_{\mathrm{AC}} R_{i \downarrow}^{\prime} \downarrow i \in\{1,2\}}{\mathrm{ri}_{w}\left(R_{1}^{\prime}, R_{2}^{\prime}\right) \in \mathcal{H}_{\mathrm{e}}(K)} \\
\text { EQ. CONSEQ. } \frac{\mathrm{k}_{w}(R, t) \in \mathcal{H}(K) \quad \mathrm{i}_{w}\left(R, R^{\prime}\right) \in \mathcal{H}_{\mathrm{e}}(K)}{\mathrm{k}_{w}\left(R^{\prime}, t\right) \in \mathcal{H}_{\mathrm{e}}(K)}
\end{gathered}
$$

Fig. 7: Rules of $\mathcal{H}_{\mathrm{e}}(K)$

\section{Soundness and completeness}

We shall show that the solved statements of any $K \in \operatorname{sat}\left(K_{\text {init }}(P)\right)$ form a complete abstraction of $P$, with respect to some extension of $\mathcal{H}$ that we define next.

Definition 15: Let $K$ be a set of statements. We define $\mathcal{H}_{\mathrm{e}}(K)$ to be the smallest set of ground facts containing $\mathcal{H}(K)$ and that is closed under the rules of Figure 7.

Theorem 2: Let $K \in \operatorname{sat}\left(K_{\text {init }}(P)\right)$ for some ground process $P$. We have that $P \models f$ for any $f \in K \cup \mathcal{H}_{\mathrm{e}}(K)$ and, if $(P, \emptyset) \stackrel{L_{1}, \ldots, L_{n}}{\longrightarrow}(Q, \varphi)$, then:

1) $\mathrm{r}_{L_{1} \varphi \downarrow, \ldots, L_{n} \varphi \downarrow} \in \mathcal{H}_{\mathrm{e}}\left(K_{\text {solved }}\right)$;

2) if $\varphi \vdash_{R} t$ then $\mathrm{k}_{L_{1} \varphi \downarrow, \ldots, L_{n} \varphi \downarrow}(R, t \downarrow) \in \mathcal{H}_{\mathrm{e}}\left(K_{\text {solved }}\right)$; 
3) if $\varphi \vdash_{R} t$ and $\varphi \vdash_{R^{\prime}} t$, then $\mathrm{i}_{L_{1} \varphi \downarrow, \ldots, L_{n} \varphi \downarrow}\left(R, R^{\prime}\right) \in$ $\mathcal{H}_{\mathrm{e}}\left(K_{\text {solved }}\right)$.

The proof follows the same general outline as in the original argument without xor [19]. However, some points that were irrelevant or straightforward in the original proof require special attention here.

As explained before, our marking discipline is justified by means of rearrangements of CONSEQ rules with $f_{0}^{+}$statements in derivation trees of $\mathcal{H}(K)$. Due to these rearrangements, inductions on derivation trees are not straightforward anymore. We sometimes have to prove the existence of a derivation tree which is smaller only for a complex measure, where in the original proof a standard induction on the size of the derivation tree or on the size of the recipe of the head was sufficient. The existence of these other derivation trees themselves relies on invariants of the saturation procedure that we enforce by the canonization rules and the distinguished VIP statements. This is in sharp contrast with the original proof where the canonization rules only act as an optimization to terminate faster.

We finally note that our saturation procedure also brings new improvements that are not directly related to xor (e.g., removal of non normal terms, canonization for unsolved statements) but which we had to introduce (and justify) to obtain an effective procedure supporting xor. Discarding these unnecessary statements could also improve the efficiency of the original AKISS procedure.

\section{Algorithm}

In this section, we first discuss the effectiveness and termination of the saturation procedure, and describe our algorithm to verify trace inclusion for determinate processes.

\section{A. Effectiveness of the procedure}

The termination of the procedure has been proved for the original version of AKISS but only for subterm convergent equational theories. It is shown, among others, that the initial knowledge base only needs to be derived from finitely many seed statements, and in particular it does not need to contains all fresh nonces the attacker can generate. As stated below, this result is also true in our setting.

Lemma 1: Let $P$ be a ground process, $\mathcal{N}_{\text {pub }}^{P} \subseteq \mathcal{N}_{\text {pub }}$ be the finite set of public names occurring in $P$. We have that:

$$
\begin{aligned}
& \operatorname{sat}\left(K_{\text {init }}(P)\right) \supseteq \\
& \quad\left\{K \cup \operatorname{ext}(K) \mid K \in \operatorname{sat}\left(K_{\text {init }}\left(\operatorname{seed}\left(P, \mathcal{N}_{\text {pub }}^{P}\right)\right)\right)\right\} .
\end{aligned}
$$

where $\operatorname{ext}(K)$ is the set containing the following statements:

- $\mathrm{k}_{\epsilon}(n, n) \Leftarrow$ for any $n \in \mathcal{N}_{\text {pub }} \backslash \mathcal{N}_{\text {pub }}^{P}$;

- $\mathrm{i}_{\epsilon}(n, n) \Leftarrow$ for any $n \in \mathcal{N}_{\text {pub }} \backslash \mathcal{N}_{\text {pub }}^{P}$;

- $\operatorname{ri}_{u}(n, n) \Leftarrow B_{1}, \ldots, B_{n}$ for any $\mathrm{r}_{u} \Leftarrow B_{1}, \ldots, B_{n} \in K$ in solved form, any $n \in \mathcal{N}_{\text {pub }} \backslash \mathcal{N}_{\text {pub }}^{P}$.

Another issue is that, when computing the update operator, we need to check whether there exists $R$ such that the statement $\mathrm{k}_{w}(R, t) \Leftarrow B_{1}, \ldots B_{n}$ is a consequence of a set of solved statements. This can be achieved using a simple backward search, similar to the one in [19].

However, with the addition of the xor, the saturation procedure may itself not terminate even if the initial knowledge base is finite and each saturation step is computable. A first reason would be the use of an unsuitable selection function. In order to avoid termination issues, we will consider a selection function that selects in priority a marked literal when it exists, a literal which is not a sum otherwise, and one that contains a rigid factor as a last resort. In case there is no other choice than selecting a literal containing a sum of variables the saturation enters an infinite loop as illustrated by the following example.

Example 14: Consider the ground process:

$$
P=\operatorname{in}(c, x) \cdot \operatorname{in}(c, y) \cdot \operatorname{in}(c, z) \cdot[x=y \oplus z] .0 .
$$

Among others, the set of seed statements will contain:

$$
\mathrm{r}_{w} \Leftarrow \mathrm{k}_{w_{1}}(X, y \oplus z), \mathrm{k}_{w_{2}}(Y, y), \mathrm{k}_{w_{3}}(Z, z) \| \emptyset
$$

where $w=\operatorname{in}(c, y \oplus z) \cdot \operatorname{in}(c, y) \cdot \operatorname{in}(c, z)$.test, and for some $w_{1}, w_{2}$, and $w_{3}$ that we do not specify since they are not relevant here. The RESOLUTION+ rule will be applied on the first hypothesis, and since there is no rigid factor in $y \oplus z$, no hypothesis will be marked. We will therefore generate (among others) a new statement of the following form:

$$
\begin{aligned}
\mathrm{r} \Leftarrow & \mathrm{k}\left(X_{1}, y_{1} \oplus z_{1}\right), \mathrm{k}\left(X_{2}, y_{2} \oplus z_{2}\right), \\
& \mathrm{k}\left(Y, y_{1} \oplus y_{2}\right), \mathrm{k}\left(Z, z_{1} \oplus z_{2}\right) \| \emptyset
\end{aligned}
$$

on which the same RESOLUTION+ rule can be applied again, entering an infinite loop.

Even though this example illustrates that termination is not guaranteed, we were able to verify a large range of different protocols, as illustrated in Section VI

\section{B. Description and correctness of the algorithm}

Our procedure is described in Figure 8 Let $\mathcal{P}$ be a protocol, i.e., a finite set of processes, and $P$ be a ground process. Let $K^{0} \in \operatorname{sat}\left(K_{\text {init }}\left(\operatorname{seed}\left(P, \mathcal{N}_{\text {pub }}^{P}\right)\right)\right)$ be a saturation of $P$ where $\mathcal{N}_{\text {pub }}^{P}$ is the set containing all public names occurring in $P$. In our procedure the process $P$ will be represented by the set $K_{\text {solved }}^{0}$ of solved statements of $K^{0}$.

The test REACH-IDENTITY $\left(K_{\text {solved }}^{0}, \mathcal{P}\right)$ checks whether each reachable identity $\mathrm{ri}_{\ell_{1}, \ldots, \ell_{n}}\left(R, R^{\prime}\right) \Leftarrow B_{1}, \ldots, B_{m}$ in $K_{\text {solved }}^{0}$ holds in $\mathcal{P}$. To perform this check for a given reachable identity, we first compute the recipes $R_{i}$ that allow the process $P$ to execute the trace $\ell_{1} \sigma, \ldots, \ell_{n} \sigma$. The substitution $\sigma$ replaces variables by fresh names in order to close the run. Next we check whether the corresponding trace $\left(M_{1}, \ldots, M_{n}\right)$ is executable in $\mathcal{P}$ and whether the test $R \omega \stackrel{?}{=} R^{\prime} \omega$ holds in the resulting frame $\varphi$, i.e., the frame reached by $\mathcal{P}$ after performing $M_{1}, \ldots, M_{n}$. If all the tests succeed, $P$ is trace included in $\mathcal{P}$. If one test fails, the algorithm returns this test as a witness of non equivalence. 
$\operatorname{REACH}-\operatorname{IdENTITY}\left(K_{\text {solved }}^{0}, \mathcal{P}\right)$

For all $\mathrm{ri}_{\ell_{1}, \ldots, \ell_{n}}\left(R, R^{\prime}\right) \Leftarrow \mathrm{k}_{w_{1}}\left(X_{1}, x_{1}\right), \ldots, \mathrm{k}_{w_{m}}\left(X_{m}, x_{m}\right) \in K_{\text {solved }}^{0}$

let $c_{1}, \ldots, c_{k}$ be fresh public names such that $\sigma: \operatorname{vars}\left(\ell_{1}, \ldots, \ell_{n}\right) \cup\left\{x_{1}, \ldots, x_{m}\right\} \rightarrow\left\{c_{1}, \ldots, c_{k}\right\}$ is a bijection

for all $i$ such that $\ell_{i}=\operatorname{in}\left(d_{i}, t_{i}\right)$, let $R_{i}$ be such that $\mathrm{k}_{\ell_{1} \sigma, \ldots, \ell_{i-1} \sigma}\left(R_{i}, t_{i} \sigma\right) \in \mathcal{H}\left(K_{\text {solved }}^{0} \cup\left\{\mathrm{k}_{\epsilon}\left(c_{i}, c_{i}\right) \Leftarrow \mid 1 \leq i \leq k\right\}\right)$

let $M_{i}= \begin{cases}\ell_{i} & \text { if } \ell_{i} \in\{\operatorname{test}, \boldsymbol{o u t}(c) \mid c \in \mathcal{C} h\} \\ \mathbf{i n}\left(d_{i}, R_{i}\right) & \text { if } \ell_{i}=\operatorname{in}\left(d_{i}, t_{i}\right)\end{cases}$

check that $(\mathcal{P}, \emptyset) \stackrel{M_{1}, \ldots, M_{n}}{\longrightarrow}(P, \varphi)$ and $R \omega \varphi \downarrow={ }_{\mathrm{AC}} R^{\prime} \omega \varphi \downarrow$ where $\omega=\left\{X_{i} \mapsto x_{i} \sigma\right\}$.

Fig. 8: Test for checking $P \sqsubseteq \mathcal{P}$

Theorem 3: Let $P$ be a ground process, and $\mathcal{N}_{\text {pub }}^{P} \subseteq \mathcal{N}_{\text {pub }}$ be the finite set of public names occurring in $P$. Let $\mathcal{P}$ be a protocol, and $K^{0} \in \operatorname{sat}\left(K_{\text {init }}\left(\operatorname{seed}\left(P, \mathcal{N}_{\text {pub }}^{P}\right)\right)\right)$. We have that:

- if $P \sqsubseteq \mathcal{P}$ then Reach-Identity $\left(K_{\text {solved }}^{0}, \mathcal{P}\right)$ holds;

- if $\mathcal{P}$ is determinate and $\operatorname{ReACH}-\operatorname{IDENTITY}\left(K_{\text {solved }}^{0}, \mathcal{P}\right)$ holds then $P \sqsubseteq \mathcal{P}$.

Note that REACH-IDENTITY requires to compute, for each input of the run, a recipe $R_{i}$. It necessarily exists (as the run $\ell_{1}, \ldots, \ell_{n}$ is reachable) and its computation amounts to finding $R$ such that $\mathrm{k}_{w}(R, t) \in \mathcal{H}(K)$ given $w, t, K$. This can be achieved using a simple recursive backward search, as in [19].

\section{IMPLEMENTATION AND CASE STUDIES}

Given that our procedure may not terminate, our main goal was to evaluate whether termination is achieved in practice. We validate our approach by integrating our procedure in the tool AKISS [3] and by testing it on various examples.

\section{A. Integration in AKISS}

Our implementation includes the marking strategy, a selection function that returns in priority an hypothesis that is marked and reasoning modulo AC. In general, it is difficult to implement unification and computation of variants for theories involving an $\mathrm{AC}$ operator. We leverage an existing tool, namely Maude [36], which implements such algorithms efficiently. To minimize the high cost of external calls to Maude, we also implement a naive algorithm for AC unification that handles most of the simple cases internally, and we only call Maude for solving the difficult cases ( $\sim 3 \%$ of cases in practice).

To ease the specification of the protocols our tool supports additional operators in the process calculus for parallel composition $(P \| Q)$, non-deterministic choice $(P++Q)$, sequential composition $(P:: Q)$, and a phase operator $(P \gg Q)$. The latter allows at any moment during the execution of $P$ to proceed to the execution of $Q$ and will be used among others to model resistance against guessing attacks as done e.g. in the ProVerif tool [17]. All these operators are syntactic sugar and can be translated to sets of (linear) processes in a straightforward way.

To mitigate the potential exponential blowup caused by this translation, partial order reduction techniques [9], [10] can be used. We integrate some of these optimisations which notably consist in automatically prioritising outputs when protocols are determinate [10].
Note that for AKISS finding attacks or proving their absence are equally difficult tasks, as for both it first completes the saturation of the traces. However, for some equivalence properties, one of the inclusions is trivially satisfied. In such a case, we only check the inclusion that is not trivially satisfied and therefore reduce by two the number of symbolic traces to explore. Note that our procedure easily allows to check inclusion instead of equivalence.

We now report on experimental results that have been obtained by running our tool on a 20 core $\operatorname{Intel}(\mathrm{R}) \operatorname{Xeon}(\mathrm{R})$ @ $3.10 \mathrm{GHz}$ with $300 \mathrm{~GB}$ of RAM.

\section{B. Unlinkability of RFID protocols}

We analyse an unlinkability property on various RFID protocols that rely on the xor operator. A description of these protocols can be found in [42], [37], [7], and one of them, namely the KCL protocol, is detailed in Example 4.

We model unlinkability as an interaction between the reader and either $\operatorname{tag}_{1}$ or $\operatorname{tag}_{2}$ assuming that the attacker has previously observed a session between the reader and $\operatorname{tag}_{1}$. We use the process $P_{\text {tag }}$ (Example 4 to model the tag. The processes $P_{\mathrm{tag}_{1}}$ and $P_{\mathrm{tag}_{2}}$ are slightly different versions of $P_{\mathrm{tag}}$. More precisely, they are obtained from $P_{\text {tag }}$ by replacing $i d$ and $k$ by $i d_{1}$ and $k_{1}$ (resp. $i d_{2}$ and $k_{2}$ ). Then, we consider a process $P_{\text {init }}$ to model the outputs observed by an attacker during an honest interaction between $\operatorname{tag}_{1}$ and the reader:

$$
P_{\text {init }}=\operatorname{out}(c, r) \text {. out }\left(c,\left\langle i d_{1} \oplus r^{\prime}, \mathrm{h}\left(\left\langle r, k_{1}\right\rangle\right) \oplus r^{\prime}\right\rangle\right) \text {. } 0
$$

Using non-deterministic choice we model that the reader may engage a session with $\operatorname{tag}_{1}$ (using $i d_{1}$ and key $k_{1}$ ) or $\operatorname{tag}_{2}$ (using $i d_{2}$ and key $k_{2}$ ):

$$
\begin{aligned}
& P_{\text {reader }}=\operatorname{out}\left(c, r_{1}\right) . \mathbf{i n}(c, y) . \\
& \quad\left(\left[\left(\operatorname{proj}_{1}(y) \oplus i d\right) \oplus \operatorname{proj}_{2}(y)=\mathrm{h}\left(\left\langle r_{1}, k_{1}\right\rangle\right)\right] . \mathbf{0}\right) \\
& ++\left(\left[\left(\operatorname{proj}_{1}(y) \oplus i d\right) \oplus \operatorname{proj}_{2}(y)=\mathrm{h}\left(\left\langle r_{1}, k_{2}\right\rangle\right)\right] . \mathbf{0}\right)
\end{aligned}
$$

Unlinkability can finally be expressed as the following process equivalence:

$$
P_{\text {init }}::\left(P_{\text {tag }_{1}} \| P_{\text {reader }}\right) \approx P_{\text {init }}::\left(P_{\text {tag }_{2}} \| P_{\text {reader }}\right)
$$

The (known) attack explained in Example 8 on a simplified scenario (without the readers) is again found by the tool. A possible fix would be to replace the message $\mathrm{h}\left(\left\langle r_{1}, k\right\rangle\right) \oplus r_{2}$ by $\mathrm{h}\left(\left\langle r_{1}, k \oplus r_{2}\right\rangle\right)$. Our tool is then able to establish unlinkability.

In total we modelled 7 RFID protocols: KCL, LD, LAK, OTYT and YPL from [42]; MW from [37]; and AT from [7]. 
Note that one inclusion trivially holds: when considering two different tags less equalities hold than in the case of two identical tags. We can therefore avoid unnecessary computations and only check the other inclusion, i.e.:

$$
P_{\text {init }}::\left(P_{\mathrm{tag}_{1}} \| P_{\text {reader }}\right) \sqsubseteq P_{\text {init }}::\left(P_{\operatorname{tag}_{2}} \| P_{\text {reader }}\right)
$$

On 4 of the 7 protocols we find (known) attacks which violate unlinkability. The results are summarised in Figure 9 and confirm termination of the saturation procedure with our resolution strategy when analysing unlinkability on various RFID protocols. When there is no attack, we consider an additional scenario where the attacker can abort its first observation to start a new session:

$$
\begin{aligned}
& P_{\text {init }}::\left(\left(P_{\operatorname{tag}_{1}} \| P_{\text {reader }}\right) \gg\left(P_{\operatorname{tag}_{1}} \| P_{\text {reader }}\right)\right) \\
\approx & P_{\text {init }}::\left(\left(P_{\operatorname{tag}_{2}} \| P_{\text {reader }}\right) \gg\left(P_{\operatorname{tag}_{2}} \| P_{\text {reader }}\right)\right)
\end{aligned}
$$

\begin{tabular}{|l|c|r|r|c|}
\hline RFID Protocol & \# sessions & \# traces & time & result \\
\hline KCL (Ex. 4 & 1 & 1 & $1 \mathrm{~s}$ & $\times$ \\
KCL & 1 & 20 & $3 \mathrm{~s}$ & $\times$ \\
KCL fixed & 1 & 20 & $<1 \mathrm{~s}$ & $\checkmark$ \\
KCL fixed & 2 & 980 & $1 \mathrm{~m} 47$ & $\checkmark$ \\
\hline LD & 1 & 20 & $<1 \mathrm{~s}$ & $\times$ \\
OTYT & 1 & 20 & $<1 \mathrm{~s}$ & $\times$ \\
YPL & 1 & 20 & $<1 \mathrm{~s}$ & $\times$ \\
LAK & 1 & 20 & $<1 \mathrm{~s}$ & $\checkmark$ \\
LAK & 2 & 980 & $30 \mathrm{~m}$ & $\checkmark$ \\
MW & 1 & 40 & $7 \mathrm{~s}$ & $\checkmark$ \\
MW & 2 & 4280 & $10 \mathrm{~h}$ & $\checkmark$ \\
AT & 1 & 20 & $1 \mathrm{~s}$ & $\checkmark$ \\
AT & 2 & 1040 & $33 \mathrm{~s}$ & $\checkmark$ \\
\hline
\end{tabular}

We note $\checkmark$ when AKISS concludes that the property holds and $X$ when it reports an attack.

Fig. 9: Summary for RFID protocols

\section{Resistance against offline guessing attacks}

We analyse resistance against offline guessing attacks on various password based protocols that rely on the exclusive-or operator from [32]. Protocols which rely on passwords are often subject to off-line guessing attacks: an attacker may observe, or even actively participate in an execution of the protocol and then, in a second, offline phase, verify if a guessed value is indeed the real password without further interaction with the protocol. During this offline verification phase, the attacker has to try all possible values for the password and perform a test to check whether a guess is the real password or not. This can be modelled relying on trace equivalence by checking whether the attacker can make a difference between a scenario where the real password is revealed at the end, and another one where a wrong password is revealed [25], [13].

We illustrate this encoding on the so-called direct authentication protocol [32] whose description is detailed below.

Direct authentication protocol. $A$ and $B$ initially share a poorly chosen secret $p w$, and wish to establish a session key $k$.
To achieve this goal, $A$ generates a public key $p u b$ and sends it to $B$ encrypted with $p w$ together with a challenge $r a$. $B$ replies by computing a ciphertext that contains fresh nonces $n b_{1}$, $n b_{2}$, and another one $c b$ called a confounder. This ciphertext also contains an encryption of the challenge $r a$ with $p w$ for authentication purposes. Then, $A$ generates a fresh key $k$, and a challenge response mechanism is used to ensure that both parties agree on the key.

$$
\begin{array}{ll}
A \rightarrow B: & r a,\{p u b\}_{p w} \\
B \rightarrow A: & \left\{B, A, n b_{1}, n b_{2}, c b,\{r a\}_{p w}\right\}_{p u b} \\
A \rightarrow B: & \left\{n b_{1}, k \oplus n b_{2}\right\}_{p w} \\
B \rightarrow A: & \left\{f_{1}(r a), r b\right\}_{k} \\
A \rightarrow B: & \left\{f_{2}(r b)\right\}_{k}
\end{array}
$$

This protocol has been designed to be resistant against guessing attacks. An attacker should not be able to perform an off-line verification of whether a guess of the password is successful or not. Actually, several examples of such protocols are described in [32]. They try to achieve the same goal relying on slightly different primitives or considering a different environment (e.g. some of them rely on a trusted third party $S$ ).

We model resistance against guessing attacks by checking an equivalence property between a scenario where the real password is revealed at the end, and another where a wrong password (modeled through a fresh name) is revealed [25], [13]. For each protocol, we consider one session between two honest agents $A$ and $B$ (and the trusted third party $S$ when needed). Resistance against guessing attacks is expressed through the following equivalence:

$$
\left(P_{A} \| P_{B}\right) \gg \operatorname{out}(c, p w) \approx\left(P_{A} \| P_{B}\right) \gg \operatorname{out}\left(c, p w^{\prime}\right)
$$

where $p w$ is the real password, and $p w^{\prime}$ is the fresh name.

In total, we modelled 5 password-based protocols: the Toy, Nonce, Secret Public Key, and Direct Authentication protocols from [32], as well as a protocol by Gong [31]. For each protocol we first verify resistance against guessing attacks in the presence of a passive adversary, i.e. a pure eavesdropper. Whenever this equivalence holds, we analyse the active case for one session. Since one inclusion trivially holds, we only check the other one, i.e.:

$$
\left(P_{A} \| P_{B}\right) \gg \operatorname{out}(c, p w) \sqsubseteq\left(P_{A} \| P_{B}\right) \gg \operatorname{out}\left(c, p w^{\prime}\right)
$$

The results are summarised in Figure 10 and illustrate that our tool can also be effectively used to analyse resistance against guessing attacks on various password-based protocols. The Direct Auth protocol has been tested with two instances of each role in parallel, the other protocols (Nonce and Sec Pub Key) require more than $50 \mathrm{~Gb}$ of memory and have not been successfully tested.

\section{Other case studies}

We have also encoded some authentication properties as equivalences for both RFID protocols that guarantee unlinkability (the LAK and fixed KCL protocols) and for a xor-based variant of the NSL protocol [22]. For both LAK and NSL-Xor 


\begin{tabular}{|l|c|c||r|r|r|c|}
\hline \multirow{2}{*}{} & \multicolumn{2}{|c||}{ passive case } & \multicolumn{4}{c|}{ active case } \\
\cline { 2 - 7 } & time & result & \#sessions & \# tr. & time & result \\
\hline Toy & $<1 \mathrm{~s}$ & $\checkmark$ & 1 & 5 & $<1 \mathrm{~s}$ & $\times$ \\
Nonce & $<1 \mathrm{~s}$ & $\checkmark$ & 1 & 90 & $4 \mathrm{~s}$ & $\checkmark$ \\
Sec PubKey & $<1 \mathrm{~s}$ & $\checkmark$ & 1 & 90 & $4 \mathrm{~s}$ & $\checkmark$ \\
Direct Auth & $<1 \mathrm{~s}$ & $\checkmark$ & 1 & 29 & $<1 \mathrm{~s}$ & $\checkmark$ \\
& & & 2 & $18 \mathrm{k}$ & $9 \mathrm{~m}$ & $\checkmark$ \\
Gong & $26 \mathrm{~s}$ & $\times$ & \multicolumn{4}{c}{ not relevant } \\
\hline
\end{tabular}

We note $\checkmark$ when AKISS concludes that the property holds and $X$ when it reports an attack.

Fig. 10: Summary for password-based protocols

we are able to find (known) attacks. The attack on NSL-xor is a variant of Lowe's classical man in the middle attack which is prevented on NSL, but possible on NSL-xor. To find the attack we analyse a scenario where $A$ starts a session with the attacker and $B$ a session with $A$.

Finally, on the NSL-xor protocol we verified strong secrecy of the nonces $n_{a}$ and $n_{b}$, as defined by Blanchet [15]: the adversary initially provides two values and must be unable to distinguish the situations where the first, respectively, the second value is used as the secret. For instance strong secrecy of the nonce $n_{a}$ is modelled as follows.

$$
\begin{aligned}
\operatorname{in}\left(c, x_{1}\right) \cdot \operatorname{in}\left(c, x_{2}\right) & :: \operatorname{NSL}\left\{x_{1} / n_{a}\right\} \\
\mathbf{i n}\left(c, x_{1}\right) \cdot \operatorname{in}\left(c, x_{2}\right) & :: \operatorname{NSL}\left\{x^{x_{2}} / n_{a}\right\}
\end{aligned}
$$

We show that neither $n_{a}$ nor $n_{b}$ are strongly secret, even when we only consider one honest session among $A$ and $B$. The results are summarised in Figure 11.

\begin{tabular}{|l|c|c|c|c|}
\hline Protocol & \# sessions & \# traces & time & result \\
\hline LAK auth & 1 & 1 & $<1 \mathrm{~s}$ & $\times$ \\
\hline KCL fixed auth & 1 & 1 & $<1 \mathrm{~s}$ & $\checkmark$ \\
KCL fixed auth & 2 & 36 & $3 \mathrm{~s}$ & $\checkmark$ \\
\hline NSL xor & 1 & 3 & $<1 \mathrm{~s}$ & $\times$ \\
$\quad$-auth & 1 & 6 & $9 \mathrm{~s}$ & $\times$ \\
-strong secrecy $n_{a}$ & 1 & 6 & $<1 \mathrm{~s}$ & $\times$ \\
-strong secrecy $n_{b}$ & 1 & & & \\
\hline
\end{tabular}

We note $\checkmark$ when AKISS concludes that the property holds and $\times$ when it reports an attack.

Fig. 11: Summary for other protocols

\section{CONCLUSION}

We presented what we believe is the first effective procedure to verify equivalence properties for protocols that use xor. The need for such verification techniques is among others motivated by the unlinkability property in RFID protocols. Our procedure builds on the theory underlying the AKISS tool and presents a novel resolution strategy which we show to be complete. Even though termination is not guaranteed the tool did terminate on all practical examples that we have tested.

Directions for future work include adding new canonization rules to extend the termination proof of AKISS [19] to theories including the xor operator. Another direction is to consider other AC operators such as Diffie-Hellman exponentiation and bilinear pairings, which are supported by the Tamarin tool [39].

\section{REFERENCES}

[1] M. Abadi and C. Fournet. Mobile values, new names, and secure communication. In 28th Symposium on Principles of Programming Languages (POPL'01), pages 104-115. ACM, 2001.

[2] M. Abadi and A. D. Gordon. A calculus for cryptographic protocols: The spi calculus. Inf. Comput., 148(1):1-70, 1999.

[3] AKISS. https://github.com/akiss/akiss

[4] M. Arapinis, T. Chothia, E. Ritter, and M. D. Ryan. Analysing unlinkability and anonymity using the applied pi calculus. In 23rd Computer Security Foundations Symposium (CSF'10), pages 107-121. IEEE Comp. Soc., 2010.

[5] A. Armando, R. Carbone, L. Compagna, J. Cuellar, and L. T. Abad. Formal analysis of SAML 2.0 web browser single sign-on: Breaking the SAML-based single sign-on for Google apps. In 6th Workshop on Formal Methods in Security Engineering (FMSE'08), pages 1-10. ACM, 2008.

[6] A. Armando et al. The AVISPA tool for the automated validation of internet security protocols and applications. In 17th International Conference on Computer Aided Verification (CAV'05), LNCS, pages 281-285. Springer, 2005.

[7] M. Asadpour and M. Torabi Dashti. A privacy-friendly rfid protocol using reusable anonymous tickets. In 10th International Conference on Trust, Security and Privacy in Computing and Communications, pages 206-213. IEEE Comp. Soc., 2011.

[8] D. Baelde, S. Delaune, I. Gazeau, and S. Kremer. Symbolic verification of privacy-type properties for security protocols with XOR (extended version). Research report, Inria Nancy - Grand Est, 2017. https://hal. inria.fr/hal-01533694

[9] D. Baelde, S. Delaune, and L. Hirschi. A reduced semantics for deciding trace equivalence using constraint systems. In 3rd International Conference on Principles of Security and Trust (POST'14), volume 8414 of $L N C S$, pages 1-21. Springer, 2014.

[10] D. Baelde, S. Delaune, and L. Hirschi. Partial order reduction for security protocols. In 26th International Conference on Concurrency Theory (CONCUR'15), volume 42 of LIPICS, pages 497-510. LeibnizZentrum für Informatik, 2015.

[11] D. A. Basin, C. Cremers, and S. Meier. Provably repairing the ISO/IEC 9798 standard for entity authentication. Journal of Computer Security, 21(6):817-846, 2013.

[12] D. A. Basin, J. Dreier, and R. Sasse. Automated symbolic proofs of observational equivalence. In 22nd Conference on Computer and Communications Security (CCS'15), pages 1144-1155. ACM, 2015.

[13] M. Baudet. Deciding security of protocols against off-line guessing attacks. In 12th Conference on Computer and Communications Security (CCS'05), pages 16-25. ACM, 2005.

[14] K. Bhargavan, A. Delignat-Lavaud, C. Fournet, A. Pironti, and P.Y. Strub. Triple handshakes and cookie cutters: Breaking and fixing authentication over TLS. In 35th Symposium on Security and Privacy (S\&P'14), pages 98-113. IEEE Comp. Soc., 2014.

[15] B. Blanchet. Automatic proof of strong secrecy for security protocols. In Symposium on Security and Privacy (S\&P'04), pages 86-100. IEEE Comp. Soc., 2004.

[16] B. Blanchet, M. Abadi, and C. Fournet. Automated Verification of Selected Equivalences for Security Protocols. In Symposium on Logic in Computer Science (LICS'05), pages 331-340. IEEE Comp. Soc., 2005.

[17] B. Blanchet, B. Smyth, and V. Cheval. Automatic Cryptographic Protocol Verifier, User Manual and Tutorial, 2016.

[18] M. Bruso, K. Chatzikokolakis, and J. den Hartog. Analysing unlinkability and anonymity using the applied pi calculus. In 23rd Computer Security Foundations Symposium (CSF'10), pages 107-121. IEEE Comp. Soc., 2010.

[19] R. Chadha, V. Cheval, Ş. Ciobâcă, and S. Kremer. Automated verification of equivalence properties of cryptographic protocol. ACM Transactions on Computational Logic, 23(4), 2016.

[20] V. Cheval, H. Comon-Lundh, and S. Delaune. Trace equivalence decision: Negative tests and non-determinism. In 18th Conference on Computer and Communications Security (CCS'11), pages 321-330. ACM, 2011. 
[21] V. Cheval, V. Cortier, and S. Delaune. Deciding equivalence-based properties using constraint solving. Theoretical Computer Science, 492:1-39, 2013.

[22] Y. Chevalier, R. Küsters, M. Rusinowitch, and M. Turuani. An NP decision procedure for protocol insecurity with XOR. In 18th Symposium on Logic in Computer Science (LICS'03), pages 261-270. IEEE Comp. Soc., 2003.

[23] H. Comon-Lundh and S. Delaune. The finite variant property: How to get rid of some algebraic properties. In Proceedings of the 16th International Conference on Rewriting Techniques and Applications (RTA'05), volume 3467 of LNCS, pages 294-307. Springer, 2005.

[24] H. Comon-Lundh and V. Shmatikov. Intruder deductions, constraint solving and insecurity decision in presence of exclusive or. In 18th Symposium on Logic in Computer Science (LICS'03), pages 271-280. IEEE Comp. Soc., 2003.

[25] R. Corin, J. Doumen, and S. Etalle. Analysing password protocol security against off-line dictionary attacks. Electr. Notes Theor. Comput. Sci., 121:47-63, 2005.

[26] V. Cortier, S. Delaune, and P. Lafourcade. A survey of algebraic properties used in cryptographic protocols. Journal of Computer Security, 14(1):1-43, 2006.

[27] S. Delaune, S. Kremer, and D. Pasaila. Security protocols, constraint systems, and group theories. In 6th International Joint Conference on Automated Reasoning (IJCAR'12), volume 7364 of LNAI, pages 164 178. Springer, 2012.

[28] S. Delaune, S. Kremer, and M. D. Ryan. Verifying privacy-type properties of electronic voting protocols. Journal of Computer Security, 17(4):435-487, July 2009.

[29] S. Escobar, C. Meadows, and J. Meseguer. Maude-NPA: Cryptographic protocol analysis modulo equational properties. In Foundations of Security Analysis and Design V, volume 5705 of LNCS, pages 1-50. Springer, 2009.

[30] S. Escobar, R. Sasse, and J. Meseguer. Folding variant narrowing and optimal variant termination. J. Log. Algebr. Program., 81(7-8):898-928, 2012.

[31] L. Gong. Using one-way functions for authentication. Computer Communication Review, 19:8-11, 1989.

[32] L. Gong, T. M. A. Lomas, R. M. Needham, and J. H. Saltzer. Protecting poorly chosen secrets from guessing attacks. IEEE Journal on Selected Areas in Communications, 11(5):648-656, 1993.

[33] A. González-Burgueño, S. Santiago, S. Escobar, C. Meadows, and J. Meseguer. Analysis of the ibm cca security api protocols in maudenpa. In 1st International Conference on Security Standardisation Research (SSR'14), volume 8893 of LNCS, pages 111-130. Springer, 2014.

[34] R. Küsters and T. Truderung. Reducing protocol analysis with XOR to the xor-free case in the Horn theory based approach. J. Autom. Reasoning, 46(3-4):325-352, 2011.

[35] G. Lowe. Breaking and fixing the Needham-Schroeder public-key protocol using FDR. In Tools and Algorithms for the Construction and Analysis of Systems (TACAS'96), volume 1055 of LNCS, pages 147-166. Springer-Verlag, 1996.

[36] The Maude system. http://maude.cs.illinois.edu/

[37] D. Molnar and D. Wagner. Privacy and security in library rfid: Issues, practices, and architectures. In 11th ACM Conference on Computer and Communications Security (CCS'04), pages 210-219. ACM, 2004.

[38] S. Santiago, S. Escobar, C. Meadows, and J. Meseguer. A formal definition of protocol indistinguishability and its verification using Maude-NPA. In 10th International Workshop on Security and Trust Management (STM'14), volume 8743, pages 162-177. Springer, 2014.

[39] B. Schmidt, S. Meier, C. Cremers, and D. Basin. The TAMARIN prover for the symbolic analysis of security protocols. In 25th International Conference on Computer Aided Verification (CAV'13), volume 8044 of LNCS, pages 696-701. Springer, 2013.

[40] A. Tiu and J. Dawson. Automating open bisimulation checking for the spi-calculus. In 23rd Computer Security Foundations Symposium (CSF'10), pages 307-321. IEEE Comp. Soc., 2010.

[41] D. Unruh. The impossibility of computationally sound XOR. IACR Cryptology ePrint Archive, 2010:389, 2010.

[42] T. van Deursen and S. Radomirovic. Attacks on RFID protocols. IACR Cryptology ePrint Archive, 2008:310, 2008. 